\title{
Degenerative Erkrankungen des Vorfußes - Kleinzehenfehlstellungen
}

\author{
D. Arbab ${ }^{1}$, B. Bouillon ${ }^{2}$, C. Lüring ${ }^{1}$
}

${ }^{1}$ Orthopädische Klinik, Klinikum Dortmund

${ }^{2}$ Klinik für Orthopädie, Unfallchirurgie und Sporttraumatologie Köln-Merheim

Fehlstellungen der Kleinzehen können isoliert, häufiger aber im Rahmen anderer Fußdeformitäten auftreten. Die Störung der Balance stabilisierender Strukturen des Metatarsophalangealgelenks führt zu einer Veränderung der Biomechanik und letztendlich zur Ausbildung unterschiedlicher Kleinzehenfehlstellungen. Konservative Maßnahmen sind bei ausgeprägten Formen häufig nicht ausreichend, sodass in diesen Fällen die Indikation zur Operation geprüft werden sollte mit der Intention einer Schaffung physiologischer Vorfußverhältnisse.

\section{Epidemiologie}

Die genaue Prävalenz und Inzidenz von Kleinzehenfehlstellungen ist nicht bekannt. Eine steigende Zunahme der Inzidenz und Prävalenz mit dem Alter scheint gesichert und der Häufigkeitsgipfel zwischen dem 60. und 70. Lebensjahr zu liegen. Frauen sind mit einem Verhältnis von 4-5: 1 deutlich häufiger betroffen, wofür neben endogenen auch externe Ursachen (Schuhmode) verantwortlich gemacht werden.

Fehlstellungen der Kleinzehen können isoliert auftreten, einen oder mehrere Zehen betreffen. Häufig treten sie als eine Komponente in komplexen Vorfußdeformitäten mit Veränderungen des I. Strahls auf. Der Anteil der operativen Kleinzehenkorrekturen an allen Vorfußoperationen liegt länderabhängig zwischen 28 und 48\%.

\section{Ätiologie}

Die Ursachen für Kleinzehenfehlstellungen sind vielfältig.

- Die häufigste Ursache ist eine Spreizfußdeformität und Pathologie des I. Strahls mit Hallux-valgusFehlstellung (HV-Fehlstellung).

- Das entsprechende Schuhwerk mit hohen Absätzen und einer Enge im Vorfußbereich begünstigen eine
Valgisierung der Großzehe und die Verdrängung der Kleinzehen.

- Eine Überlänge insbesondere des II. Strahls, wie sie bei der sogenannten griechischen Fußform vorliegt, unterstützt eine Fehlstellung und damit die Ausbildung einer dauerhaften Deformität.

- Die Pathomechanik des Vorfußes kann auch durch angeborene oder erworbene komplexe Fußfehlstellungen wie den Pes planovalgus oder den Pes cavovarus bedingt sein.

- Der Knick-Senk-Fuß begünstigt eine valgische Fehlstellung der Großzehe und führt zu einer vermehrten Spannung der Flexoren. Diese tragen zu einer Beugefehlstellung der Kleinzehen bei.

\section{Abkürzungen}

$\begin{array}{ll}\text { d.-p. } & \text { dorsoplantar } \\ \text { DIP-Gelenk } & \text { distales Interphalangealgelenk } \\ \text { FDL } & \text { Flexor digitorum longus } \\ \text { HV } & \text { Hallux valgus } \\ \text { IM-Winkel } & \text { Intermetatarsalwinkel } \\ \text { K-Draht } & \text { Kirschner-Draht } \\ \text { MIS } & \text { Minimal Incision Surgery } \\ \text { MTP-Gelenk } & \text { Metatarsophalangealgelenk } \\ \text { pAVK } & \text { periphere arterielle Verschlusskrankheit } \\ \text { PIP-Gelenk } & \text { proximales Interphalangealgelenk }\end{array}$


- Der neurogene Ballenhohlfuß kann mit einer ausgeprägten Klauenzehenfehlstellung und schmerzhafter plantarer Exposition der Mittelfußköpfe (Metatarsalgie) einhergehen.

- Frakturen der Kleinzehen oder Metatarsalia mit nicht achsgerechter Konsolidierung können eine entsprechende Deformität der Zehen in allen 3 Ebenen bedingen. Auch Unterschenkelfrakturen können bei Verletzungen von Nerven und Muskeln oder nach Ausbildung eines Kompartmentsyndroms zu entsprechenden Fehlstellungen der Zehen führen.

- Degenerative oder akute Rupturen der plantaren Gelenkkapsel des MTP-Gelenks führen zu einer Instabilität desselben und sagittalen oder auch transversalen Fehlstellungen der Kleinzehen.

- Erkrankungen aus dem rheumatischen Formenkreis gehen häufig mit progredienten Vorfußpathologien und Fehlstellungen am I. Strahl und den Kleinzehen einher. Sie weisen häufig (Sub-)Luxationen in den MTP-Gelenken und Beugekontrakturen in den PIP-Gelenken auf.

- Fehlstellungen der V. Zehe im Sinne eines Digitus quintus varus sind meist angeboren, können aber durch eine Spreizfußkonfiguration und Enge im Schuhwerk begünstigt werden.

- Der störende Kleinzehenballen kann durch eine anlagebedingte bzw. traumatische fibulare Deviation des V. Metatarsale oder eine Vergrößerung des IV. und V. intermetatarsalen Raumes bedingt sein.

\section{Pathomechanik}

Die Ausbildung von Kleinzehenfehlstellungen geht mit einem Ungleichgewicht der flektierenden und extendierenden Muskeln sowie der passiven Stabilisatoren einher und resultiert in einem Stabilitätsverlust im MTP-Gelenk.

\section{- Aktive Stabilisatoren}

Aktiv stabilisierende Faktoren sind

- die langen und kurzen Extensoren,

- die langen und kurzen Flektoren sowie

- die intrinsische Muskulatur (Mm. lumbricales und interossei).

\section{- Passive Stabilisatoren}

Als passive Stabilisatoren fungieren in erster Linie

- die plantare Gelenkkapsel und

- die Kollataralbänder des MTP-Gelenks.
Der Extensor digitorum longus führt eine Dorsalextension im Grundglied aus und kann bei Neutralstellung oder Flexion im MTP-Gelenk eine Streckung im PIPGelenk bewirken.

Der Flexor digitorum longus (FDL) und brevis setzen jeweils am End- und Mittelglied an und führen eine Beugung derselbigen durch. Eine Beugung des Grundglieds können die Flektoren nicht bewirken. Diese Aufgabe erfüllen bei regelrechter Anatomie die Mm. interossei und lumbricales als intrinsische Muskeln. Ihre Sehnen setzen plantar der Bewegungsachse des MTP-Gelenks an und flektieren bei Kontraktion im MTP-Gelenk (Abb. 1).

Die plantare Platte ist der wichtigste passive Stabilisator des MTP-Gelenks.

Die plantare Platte besteht aus der plantaren Aponeurose und der plantaren Gelenkkapsel des MTP-Gelenks und setzt an der Basis der proximalen Phalanx an. Sie wird in ihrer Funktion von den Kollateralbändern unterstützt, die an der plantaren Platte und der Basis der proximalen Phalanx inserieren.

Die Imbalance zwischen intrinsischer und extrinsischer Zehenmuskulatur oder die Verletzung der passiv stabilisierenden Strukturen können ungeachtet der Ätiologie zu einem Stabilitätsverlust im MTP-Gelenk führen und in der Ausbildung einer Kleinzehenfehlstellung resultieren. Die Dorsalextension der Kleinzehe (Verdrängung bei HV, enges Schuhwerk) führt zu einer Veränderung der Rotationsachse im MTP-Gelenk und einem Überwiegen der Extensoren.

Die Hyperextension des Grundglieds mit Plantarisierung der Bewegungsachse bedingt eine funktionelle Insuffizienz der antagonisierenden intrinsischen Muskulatur. Diese kann dann keine Plantarflektion des Grundglieds oder Extension im PIP- und DIP-Gelenk bewirken. Es kann vielmehr zu einer verstärkten Dorsalextension durch Plantarisierung der Rotationsachse und Veränderung des intrinsischen Muskelzugs kommen (Intrinsicminus-Deformität).

Die Insuffizienz und die Verletzung der plantaren Platte und des Kollateralbandkomplexes können Fehlstellungen in der Sagittal- als auch der Transversalebene bedingen.

Bei der Crossover-Toe-Deformität überkreuzt häufig die II. Zehe die Großzehe. Sie ist Ausdruck einer fortgeschrittenen Insuffizienz der plantaren Platte mit kombinierter Fehlstellung und einer (Sub-)Luxation im MTP-Gelenk. 


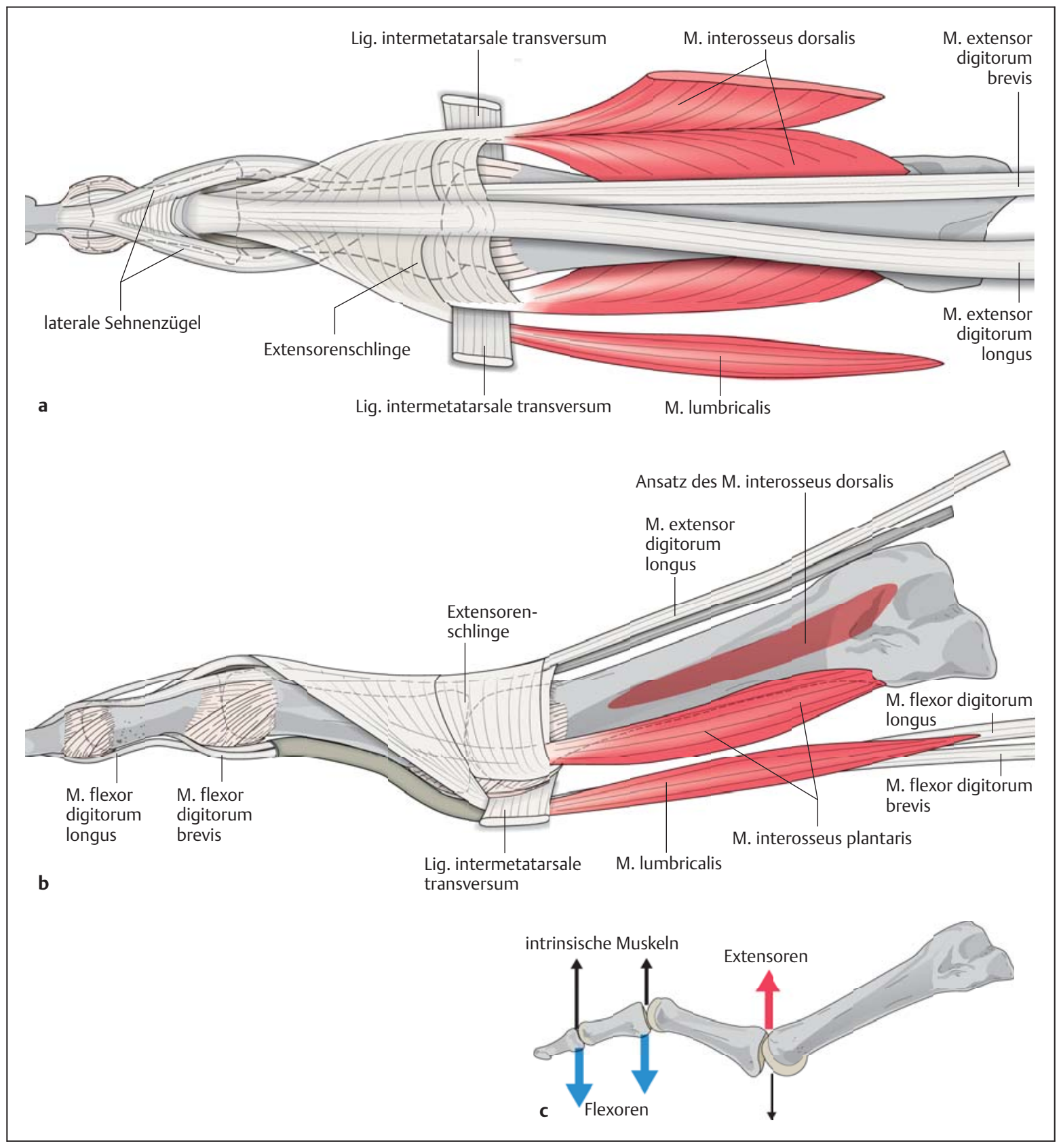

Abb. 1 - Schematische Darstellung der Anatomie der Kleinzehen von dorsal und lateral. Bei regelrechten anatomischen Verhältnissen haben die intrinsischen Muskeln (Mm. lumbricales und interossei) eine flektierende Funktion auf das Grundgelenk und der M. extensor digitorum longus eine extendierende Funktion im PIP-Gelenk. a Ansicht von dorsal. b Ansicht von lateral. c Ein Ungleichgewicht der Stabilisatoren führt zu einer Veränderung der Rotationsachse mit Überwiegen der extrinsischen Muskeln (Mm. extensor digitorum und flexor digitorum) gegenüber den intrinsischen Muskeln mit entsprechender Fehlstellung (Extension im MTP- und Flexion im PIP-Gelenk).

Die V-förmige Abspreizung der Kleinzehen wird auch als „Splay Toe“ bezeichnet. Sie resultiert meist aus einer Schwellung und relativen Enge zwischen 2 MTP-Gelenken.

\section{Klassifikation}

Fehlstellungen der Kleinzehen können sich darstellen als

- Extensions- oder Flexionsfehlstellung (sagittale Ebene),

- valgische oder varische Abweichung (transversale Ebene) oder

- als Rotationsfehlstellung (koronare Ebene). 


\section{Beckengürtel und untere Extremität}

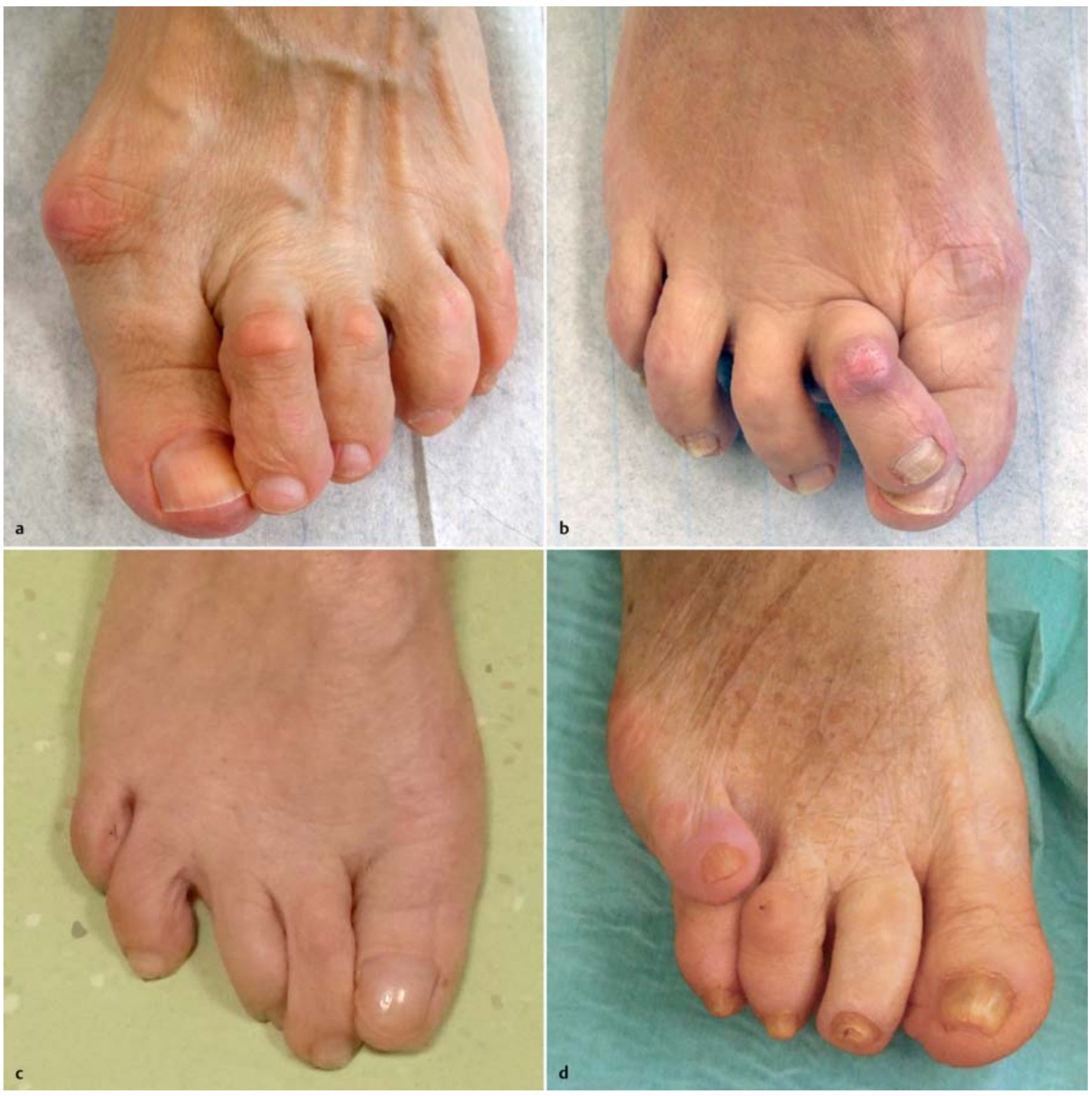

Abb. 2 - Klinische Beispiele für Fehlstellungen der Kleinzehe. a Krallen- oder Klauenzehenfehlstellung der Zehen II-IV bei Hallux-valgus-Deformität. b Crossover-Toe-Fehlstellung der II. Zehe bei Ruptur der plantaren Platte und valgischer Deformität der Großzehe. c Splay Toes der Zehen III und IV bei intermetatarsaler Enge. $\mathbf{d}$ Digitus quintus varus et superductus.

Klinische Beispiele für einige der genannten Fehlstellungen sind in Abb. 2 gezeigt, weitere sind schematisch in Abb. 3 dargestellt.

Da nicht nur im deutschsprachigen Raum eine einheitliche Nomenklatur fehlt, ist ein deskriptiver Befund der Fehlstellung für die einzelnen Gelenke (MTP-, PIP- und DIP-Gelenk) und die Ebene der Fehlstellung (valgisch/ varisch, extendiert/flektiert, innen-/außenrotiert) als auch der funktionelle Befund (flexibel/semi-/rigide, sub-/luxiert) zu bevorzugen.
Hammerzehe (Hammer Toe). Beugestellung im PIPGelenk, kann mit einer Extension im MTP-Gelenk und Beugestellung im DIP-Gelenk verbunden sein (Abb. 2 a u. Abb. 3a).

Krallen- oder Klauenzehe (Claw Toe). Extensionsstellung im MTP-Gelenk mit Beugestellung im PIP- und DIPGelenk (Abb. 3b).

Endgelenkhammerzehe (Mallet Toe). Isolierte Beugestellung im DIP-Gelenk (Abb. 3c). 


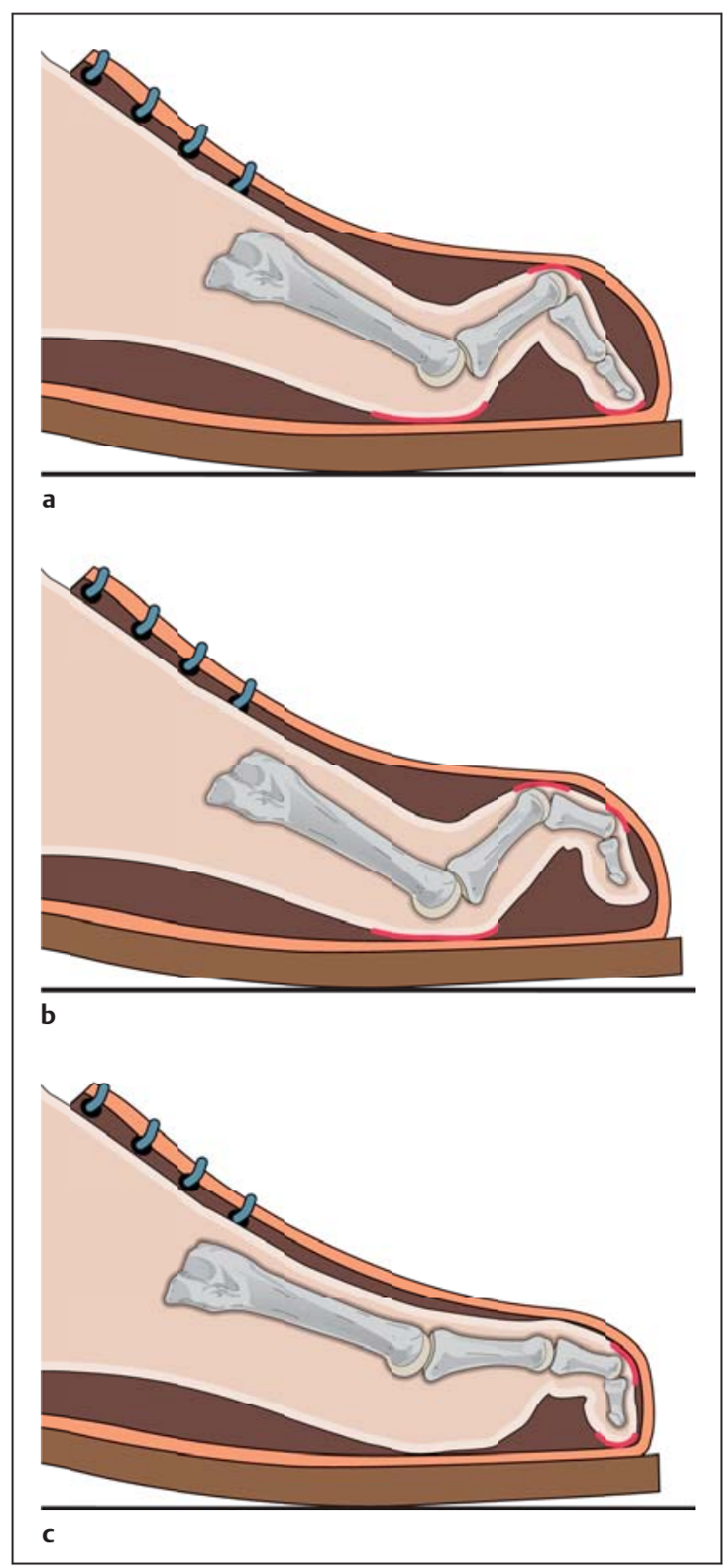

Abb. 3 - Schematische Darstellung von Zehendeformitäten. a Hammerzehe. b Krallen- oder Klauenzehe. c Endgelenkhammerzehe.

\section{Hintergrundinformation}

\section{Historisch}

Die Bezeichnung Schneiderballen oder Tailor's Bunion stammt aus dem 19. Jahrhundert, als die Schneider mit gekreuzten Beinen barfuß auf ihrem Tisch saßen und arbeiteten. Diese Haltung führte häufig zu einem schmerzhaften Druck und einer Ballenbildung über dem lateralen Metatarsale-V-Kopf.
Digitus secundus varus et superductus (Crossover Toe). Transversale und sagittale Achsabweichung im MTPGelenk mit medialer Deviation, meist der II. Zehe. Überkreuzen der betroffenen Zehe über die Großzehe, oft mit zusätzlicher Flexionsstellung im PIP-Gelenk (Abb. 2 b). Häufig in Kombination mit einer HV-Fehlstellung.

Splay Toe. Transversale Achsabweichung mit V-förmiger Abspreizung der Kleinzehen (Abb. 2c).

Kleinzehenballen. Auch als Schneiderballen, Bunionette oder Tailor's Bunion bezeichnet; Prominenz des lateralen Metatarsale-V-Kopfs (Einteilung s.Abb. 11).

Digitus quintus varus (super- oder subductus). Medial gerichtete Achsabweichung der V. Zehe mit Fehlstellung im MTP-Gelenk. Die Zehe kann sich dabei über (superductus; Abb. 2d) oder unter (subductus) die IV. Zehe einstellen.

Eine genaue Erfassung der Fehlstellungen ist für die Wahl der erforderlichen operativen Eingriffe von großer Bedeutung.

\section{Symptomatik}

Die betroffenen Patienten klagen, abhängig von der Lokalisation und Ausprägung der Kleinzehenfehlstellung, über geringe bis starke, zum Teil immobilisierende Beschwerden. Häufig liegen Pathologien des I. Strahls (HV) oder komplexe Fußdeformitäten (Pes planovalgus, Pes cavovarus) vor, die ebenfalls Beschwerden verursachen können.

Die Kleinzehen verursachen häufig belastungsabhängige Schmerzen mit entsprechendem Schuhkonflikt:

- Bei der Hammerzehe kommt es durch die Beugestellung im PIP-Gelenk zu einem dorsalseitigen Anstoßen im Schuh mit Schmerzen und ggf. Klavusbildung.

- Die Endgelenkhammerzehe zeigt häufig eine Hyperkeratose des plantaren Endglieds mit Schmerzsymptomatik und Veränderungen des Nagels.

- Bei der Krallen- oder Klauenzehe kann es durch die Extension im MTP-Gelenk zu belastungsabhängigen Schmerzen der Mittelfußköpfe (Metatarsalgie) kommen. Das dorsale PIP-Gelenk stößt, wie bei der Krallenzehe, am Oberleder der Schuhe an und verursacht entsprechende Beschwerden. 


\section{Diagnostisches Vorgehen}

\section{Klinische Untersuchung}

Die klinische Untersuchung umfasst immer beide Füße am entkleideten, zunächst stehenden Patienten. Dieses Vorgehen erlaubt eine orientierende Beurteilung von Beckenstand, Beinachsen und Stellung der Rückfüße. Die Beurteilung des Gangbilds mit und ohne Schuhwerk gestattet eine Analyse des Gangzyklus und des dynamischen Verhaltens des Fußes. Die Demonstration von differenzierten Stand- und Gangarten ermöglicht eine Beurteilung funktioneller Einschränkungen.

\section{- Inspektion}

Die Inspektion des Fußes in Belastung erfasst die Stellung der Kleinzehen, des I. Strahls sowie der Rückfußachsen. Komplexe Fußfehlstellungen müssen erfasst und mit behandelt werden, um ein dauerhaftes Korrekturergebnis der Kleinzehen zu ermöglichen. Unter Belastung sollte die Stellung der Zehen in allen 3 Ebenen analysiert werden. Die Längenverhältnisse der Zehen zueinander und ein Verlust des Bodenkontakts einzelner Zehen sollte berücksichtigt werden. Eine mögliche unbewusste Schonhaltung und unphysiologische Lastverteilung geben wertvolle Hinweise über die Lokalisation und das Ausmaß der Pathologie.

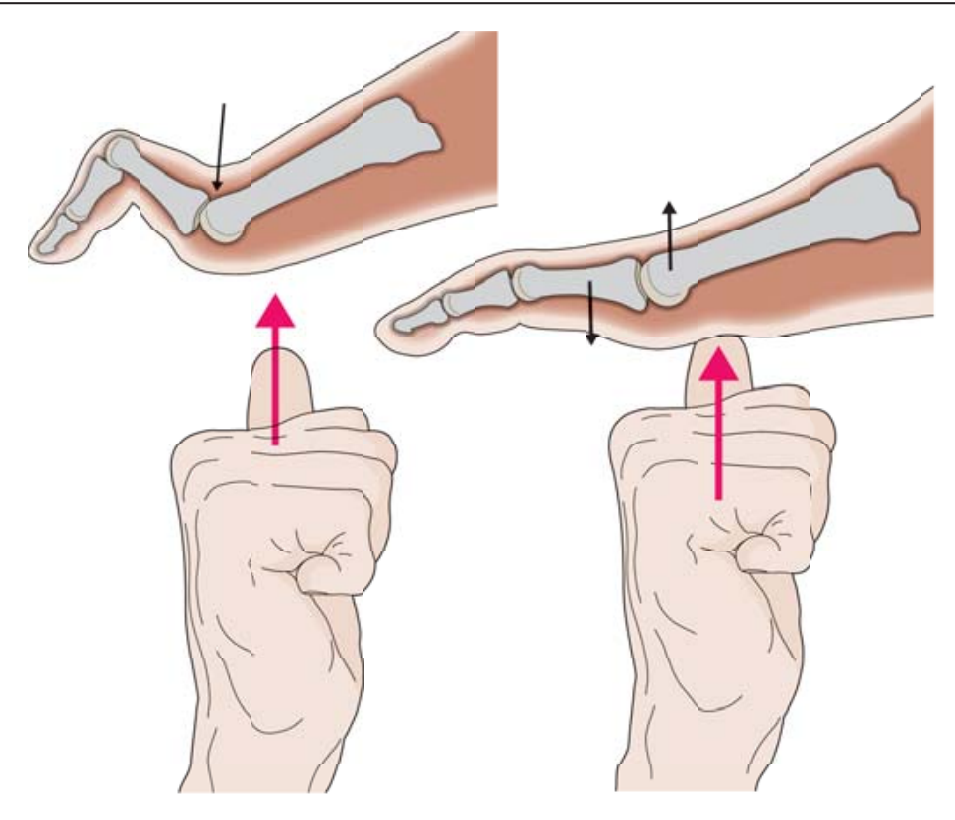

Abb. 4 - Funktionsuntersuchung Push-up-Test: Durch plantaren Druck wird eine Belastung des Vorfußes simuliert. Der Ausgleich im MTP- und PIP-Gelenk weist auf eine flexible Fehlstellung hin.
Die Inspektion des entlasteten Fußes ermöglicht eine eingehende Beurteilung der Fußsohle. Eine ausgeprägte Beschwielung ist ein Hinweis für die mechanische Überbelastung und Schmerzlokalisation insbesondere unter den Mittelfußköpfen (Metatarsalgie). Bei ausgeprägten Befunden (Klauenzehe) kann sich eine Reduzierung und Verlagerung des plantaren Fettpolsters nach distal darstellen. Endgelenkhammerzehen zeigen häufig Nagelwuchsstörungen und eine Beschwielung der Zehenkuppe. Valgische oder varische Zehenfehlstellungen können mit schmerzhaften interdigitalen Befunden einhergehen.

Sich in Entlastung korrigierende Zehenfehlstellungen lassen auf eine flexible Pathologie schließen und geben wertvolle Hinweise für das weitere therapeutische Vorgehen. Ausgeprägte persistierende Fehlstellungen lassen hingegen auch neurologische Ursachen in Betracht ziehen und müssen weiter abgeklärt werden.

\section{- Palpatorische Untersuchung}

Die palpatorische Untersuchung der Füße sollte nach einem festen Schema immer in der gleichen Weise erfolgen, um alle Pathologien systematisch zu erfassen. Für die Untersuchung des Vorfußes kann von den Tarsometatarsalgelenken über die MTP-, PIP- und DIP-Gelenke nach distal vorgegangen werden. Dabei sollten alle 5 Zehen und alle Zehengelenke untersucht werden.

\section{- Funktionsuntersuchung}

Die Funktionsuntersuchung erfasst die Beweglichkeit der Zehen und soll das Ausmaß einer passiven und aktiven Ausgleichbarkeit der Fehlstellung aufzeigen. Diese sind entscheidend für die Auswahl des entsprechenden Therapieverfahrens.

Push-up-Test. Der Push-up-Test simuliert die Belastung des Vorfußes im Stand und gibt Hinweise über das Vorliegen einer flexiblen oder nicht flexiblen Fehlstellung im MTP- und PIP-Gelenk. Über einen plantaren Druck auf Höhe der MTP-Gelenke wird die Belastung des Vorfußes aufgebaut. Korrigiert sich eine zuvor bestehende Extension der Grundphalanx mit dorsaler Subluxation im MTP-Gelenk und eine Beugestellung im PIP-Gelenk, ist von einer flexiblen Fehlstellung auszugehen (Abb.4). Ein pathologischer Push-up-Test liegt hingegen bei fixierter Fehlstellung vor.

Dieser sollte anschließend an jedem betroffenen Gelenk isoliert überprüft und dokumentiert werden. 
Schubladentest. Eine Instabilität im MTP-Gelenk hat für den Therapiealgorithmus Bedeutung und kann durch den Schubladentest erfasst werden. Dabei wird der Metatarsalkopf zwischen Daumen und Zeigefinger fixiert und die Zehe in $25^{\circ}$ Dorsalextension vom Daumen und Zeigefinger der anderen Hand vertikal bewegt (Abb. 5). Eine vermehrte dorsoplantare (d.-p.) Translation weist auf eine Instabilität als Folge einer Insuffizienz oder Verletzung der plantaren Platte hin.

\section{- Sonstige klinische Untersuchungen}

Die Erhebung eines neurologischen Untersuchungsbefunds und die Überprüfung der Perfusion sollten einhergehen mit Erfassung möglicher Infektionen und Pilzbefall der Zehen sowie Wuchsstörungen der Zehennägel. Besteht der Verdacht einer neurogenen Ätiologie, sollte eine weiterführende neurologische Untersuchung durchgeführt werden.

\section{Praxis}

\section{Zielgerichtete Diagnostik}

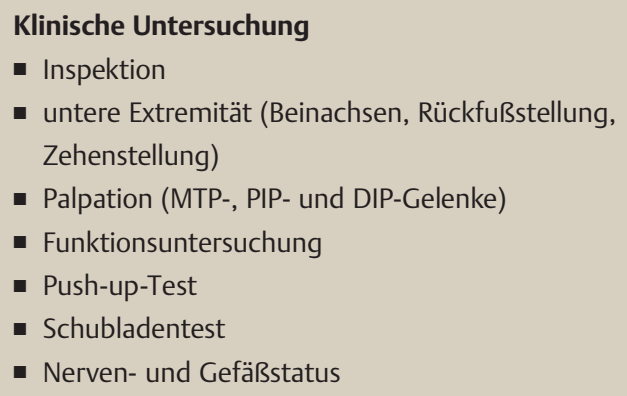

\section{Bildgebende Verfahren}

Röntgen. Auch wenn für die Festlegung des Therapieverfahrens die klinische Untersuchung von ausschlaggebender Bedeutung ist, sollten vor jeder Therapieentscheidung konventionelle Röntgenaufnahmen am belasteten Fuß im normalen beidbeinigen Stand angefertigt werden. Die d.-p. und die seitliche Aufnahme des gesamten Fußes sind für die Beurteilung der Kleinzehen i.d.R. ausreichend. Sie können aber bei weiteren Pathologien und anderen Fragestellungen durch Schrägaufnahmen ergänzt werden.

Schnittbildgebung. Eine Schnittbildgebung (Computertomografie oder Magnetresonanztomografie) ist nicht erforderlich und bleibt besonderen Fragestellungen vorbehalten.

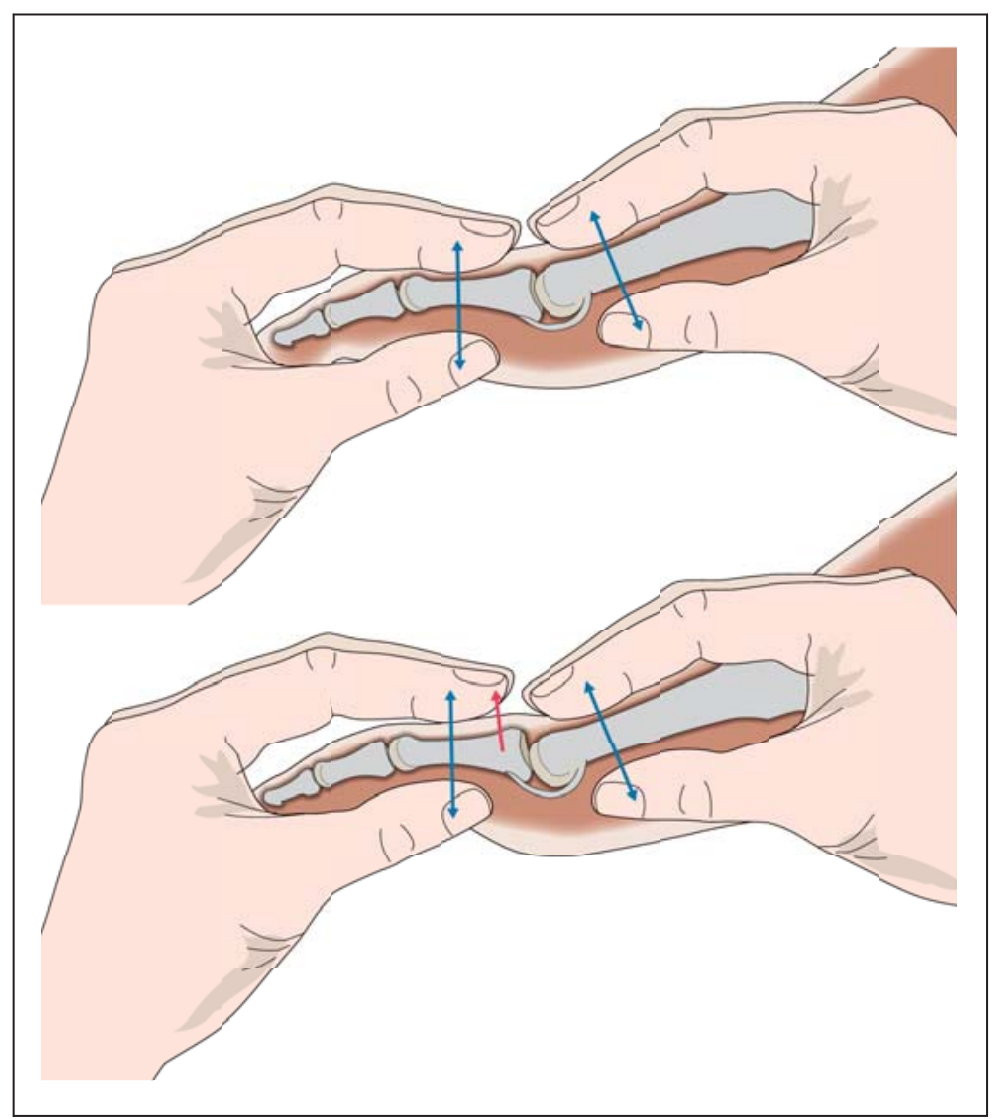

Abb. 5 - Funktionsuntersuchung Schubladentest: Die Zehe wird in leichter Extension fixiert und eine dorsoplantare Translationsbewegung durchgeführt. Eine vermehrte Translation kann Folge einer Verletzung oder Insuffizienz der plantaren Platte sein.

\section{Praxis}

\section{Zielgerichtete Diagnostik}

Röntgenbefund

- metatarsales Alignment

- dorsale (Sub-)Luxation in den MTP-Gelenken

- Intermetatarsalwinkel (II-V)

- Darstellung der Metatarsalia und Zehen (Achsabweichung, Kongruenz)

\section{Fußdruckmessung}

Die Fußdruckmessung kann ergänzende Informationen über die Lastverteilung und Belastungsspitzen liefern. Mittels Spiegelpodoskop oder Trittspurabdruck können Belastungen der Fußsohle im Stand beurteilt werden. Die moderne Pedobarografie ermöglicht darüber hinaus die dynamische Untersuchung des Abrollvorgangs und der Lastverteilung. 


\section{Therapeutisches Vorgehen}

\section{Konservative Behandlung}

Die konservative Behandlung von Kleinzehendeformitäten und der Metatarsalgie kann bei milder Fehlstellung und Schmerzen zu einer Linderung der Beschwerden und Verbesserung der Lebensqualität und Mobilität beitragen.

\section{- Schuhe}

Zunächst sind eine entsprechende Auswahl und Anpassung des Schuhwerks an die entsprechende Fußform durchzuführen. Die Schuhe sollten im Vorfußbereich eine ausreichende Breite und weiches Oberleder aufweisen. Bei Bedarf kann eine Weitung des Oberleders durchgeführt werden, um z.B. ein Anstoßen des PIP-Gelenks zu verhindern.

\section{- Einlagen}

Bei Vorliegen einer Metatarsalgie mit belastungsabhängigen Schmerzen der plantaren Mittelfußköpfe kann eine Einlagenversorgung durchgeführt werden. Die Einlagenversorgung umfasst eine individuell angepasste retrokapitale Pelotte und eine Weichbettung und Aussparung im Bereich der schmerzhaften Mittelfußköpfe. Die Modifikation der Schuhsohle im Sinne einer Schmetterlingsrolle mit Abrollhilfe kann in fortgeschrittenen Fällen und kontrakten Situationen zur Anwendung kommen.

\section{nhysiotherapie}

Mobilisierende, kräftigende und koordinative Übungen der Zehen und des Fußes können unter physiotherapeutischer Anleitung oder eigenverantwortlich durchgeführt werden. Durch die Beübung kann bei milder flexibler Fehlstellung eine Verbesserung erzielt, das Fortschreiten aber i.d.R. nicht verhindert werden.

\section{- Fußpflege}

Bei Schwielenbildung und der Gefahr der Entwicklung von Druckulzera (Diabetes mellitus) ist eine regelmäßige professionelle oder eigenverantwortliche Fußpflege zu empfehlen.

\section{- Polster}

Polsternde Druckschutzorthesen aus Schaumstoff oder Silikon können in diesen Fällen häufig Beschwerden lindern und die Ausbildung von Druckstellen verzögern.
Bei fortschreitenden und fortgeschrittenen Deformitäten lässt sich mit den genannten Maßnahmen nicht immer ein entsprechender Therapieerfolg erzielen, zudem ist die Akzeptanz insbesondere orthopädieschuhtechnischer Maßnahmen bei jüngeren und aktiven Patienten begrenzt.

\section{Operative Therapie: präoperativ zu beachten}

\section{- Indikation zur Operation}

Ein operatives Vorgehen sollte geprüft werden, wenn die o.g. konservativen Maßnahmen zu keinem entsprechenden Therapieerfolg geführt haben. Bei fortgeschrittenen Deformitäten mit ausgeprägter Klinik und Inakzeptanz orthopädieschuhtechnischer Maßnahmen ist ebenfalls ein operatives Vorgehen anzustreben.

\section{Indikationen}

\section{Operationsindikationen}

- nach Ausschöpfung konservativer Therapiemaßnahmen

- hoher Leidensdruck aufgrund von Schmerzen und Beeinträchtigung der Mobilität

- zunehmende oder ausgeprägte Deformität und Konflikt mit den übrigen Zehen

\section{- Kontraindikation}

Bei akuter Infektsituation ist von einer operativen Versorgung abzusehen. Problematische Weichteilverhältnisse und chronische Wunden an den Zehen stellen relative Kontraindikationen dar. In diesen Fällen kann die Korrektur der Fehlstellungen zu einer Verbesserung der Weichteilsituation wesentlich beitragen, wenn damit das Korrelat für die Weichteilkompromittierung beseitigt wird. Bei peripherer arterieller Verschlusskrankheit (pAVK), insulinpflichtigem Diabetes mellitus, systemischer Glukokortikoidtherapie oder Immunsuppression sollte die Indikation zur Operation zurückhaltend gestellt werden.

\section{Aufklärung}

Die allgemeinen und speziellen Risiken und Komplikationen (s. Infobox) sowie das spezielle Nachbehandlungsschema sollten in einem standardisierten Aufklärungsprotokoll dokumentiert werden. In diesem sollte auch ein befundabhängiges Vorgehen mit Erweiterung möglicher Maßnahmen abhängig vom intraoperativen Befund festgehalten werden. 
Fazit

\section{Patientenaufklärung bei Eingriffen an den Zehen II-V}

- postoperative Schwellung

- Bewegungseinschränkung

- Verletzung von Nerven und Sensibilitätsstörungen

- Fehlstellung (Malalignment)

- Über- oder Unterkorrektur

- Rezidivfehlstellung

- Wundheilungsstörung

- Pseudarthrose

- Nagelwachstumsstörungen

- Durchblutungsstörungen bis hin zur Nekrose und Amputation

- Infektion

- postoperative Ischämie und Nekrose

\section{nherapieziel}

Die Ziele der operativen Therapie sind eine dauerhafte Schmerzreduktion und orthograde Korrektur der Kleinzehenfehlstellungen. Die Achskorrektur orientiert sich an den anatomischen und biomechanischen Verhältnissen und soll eine zufriedenstellende Funktion und Kosmetik wiederherstellen.

\section{- Kriterien zur Wahl des Operationsverfahrens}

Für die operative Korrektur von Kleinzehenfehlstellungen steht eine Vielzahl von Operationstechniken zur Verfügung. Die Wahl des Operationsverfahrens ist von klinischen und radiologischen Befunden abhängig. Therapiealgorithmen können als Grundlage für das operative Vorgehen dienen. Allgemeine Faktoren, wie das Alter, der Aktivitätsanspruch, Begleiterkrankungen und der Patientenwunsch sollten aber immer eine individuelle Therapieplanung und die Anpassung der Algorithmen an die Befundkonstellation des Patienten bedingen.

\section{- Klinischer Befund}

Für die Wahl des Operationsverfahrens ist der klinische Befund von wesentlicher Bedeutung.

Nach eingehender Untersuchung und Analyse des MTP-, PIP- und DIP-Gelenks hinsichtlich Fehlstellungen und Redressierbarkeit wird eine operative Strategie festgelegt. $\mathrm{Zu}$ berücksichtigen ist immer auch eine Behandlung des I. Strahls und des Rückfußes bei entsprechender Fehlstellung.
Krallen- oder Klauenzehe. Bei der Klauenzehenfehlstellung liegt eine Subluxation oder Luxation der Zehe im MTP-Gelenk mit Extension des Grundglieds bei gleichzeitiger Beugestellung im PIP-Gelenk vor. Die manuelle Redressierbarkeit kann durch den Push-up-Test (s. o.) überprüft werden. Gelingt durch den manuellen Druck von plantar eine Reposition des MTP-Gelenks, kann ein Transfer der FDL-Sehne und ggf. ein dorsales, weichteiliges Release der Kapsel und eine Strecksehnenverlängerung ausreichend sein.

Ist durch den Push-up-Test eine manuelle Reposition nicht möglich (pathologischer Push-up-Test), sollten der dorsale Weichteileingriff und eine metatarsale Verkürzungsosteotomie durchgeführt werden. Bei begleitender kontrakter Beugefehlstellung im PIP-Gelenk ist eine achskorrigierende PIP-Arthrodese oder Resektionsarthroplastik anzuschließen. Eine dann noch persistierende Extensionsstellung im MTP-Gelenk kann durch einen Transfer der FDL-Sehne adressiert werden. Den therapeutischen Algorithmus zeigt Abb. 6.

Crossover Toe. Bei dieser Fehlstellung mit Instabilität des MTP-Gelenks und positivem Schubladentest können die oben genannten Therapiemaßnahmen bei ausbleibender Reposition mit einer Rekonstruktion der plantaren Platte kombiniert werden (Abb. 7).

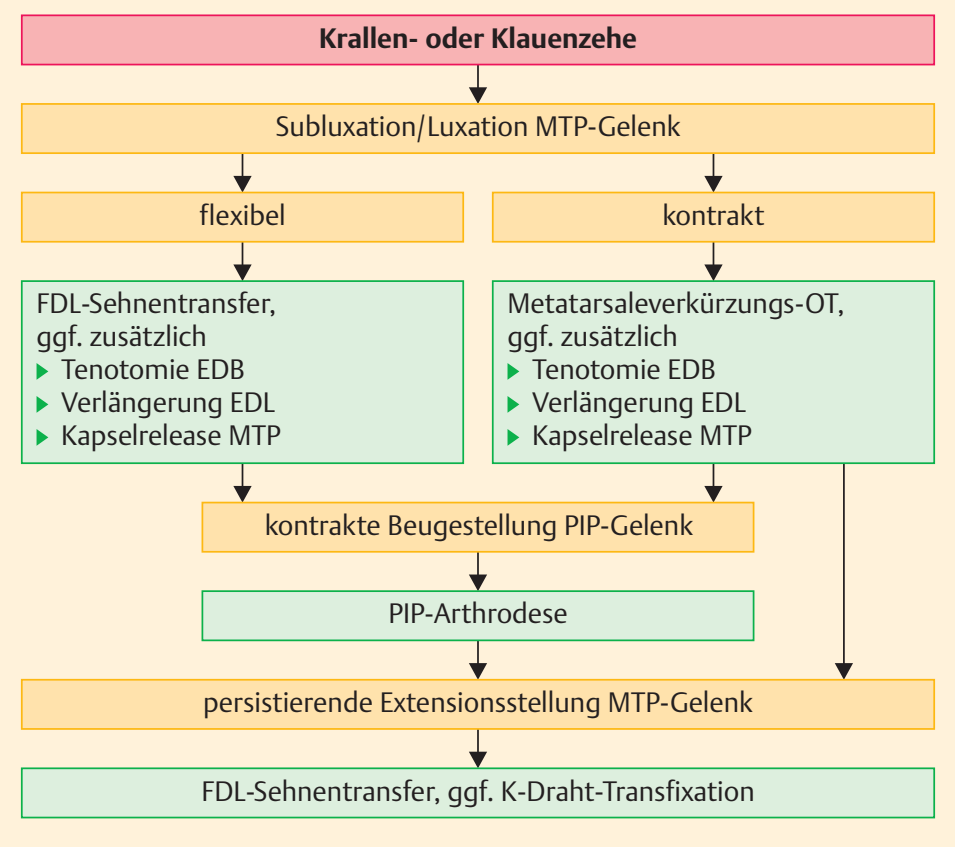

Abb. 6 - Therapeutischer Algorithmus bei der Krallen- oder Klauenzehe. EDB = Extensor digitorum brevis; $\mathrm{EDL}=$ Extensor digitorum longus; $\mathrm{FDL}=$ Flexor digitorum longus; $\mathrm{OT}=$ Osteotomie. 


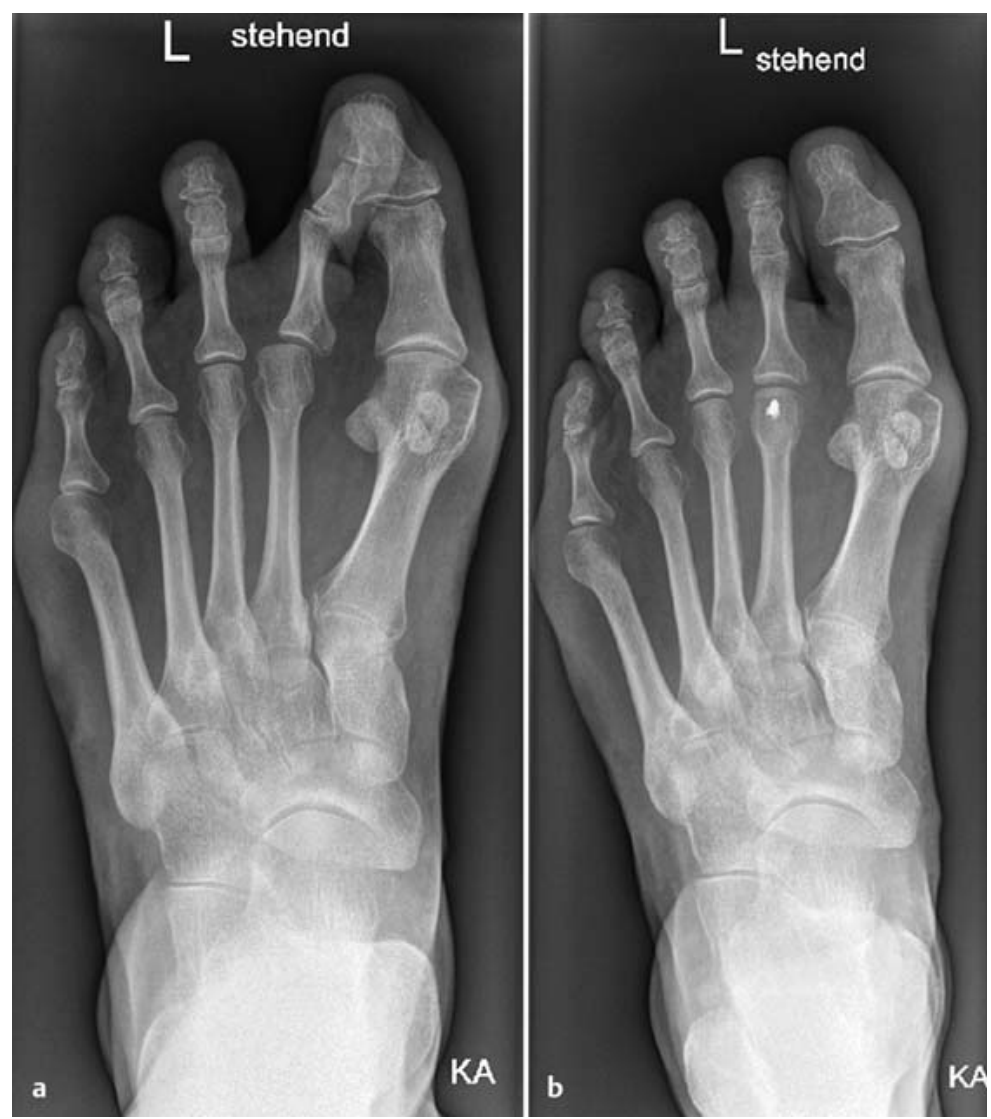

Abb. 7 - Crossover-Fehlstellung. a Röntgenbilder einer Crossover-Fehlstellung der II. Zehe bei (noch) geringer Subluxation im MTP-Gelenk. b Achsgerechte operative Korrektur der II. Zehe durch eine metatarsale Verkürzungsosteotomie nach Weil und einem Flexor-digitorum-longus-Sehnentransfer.

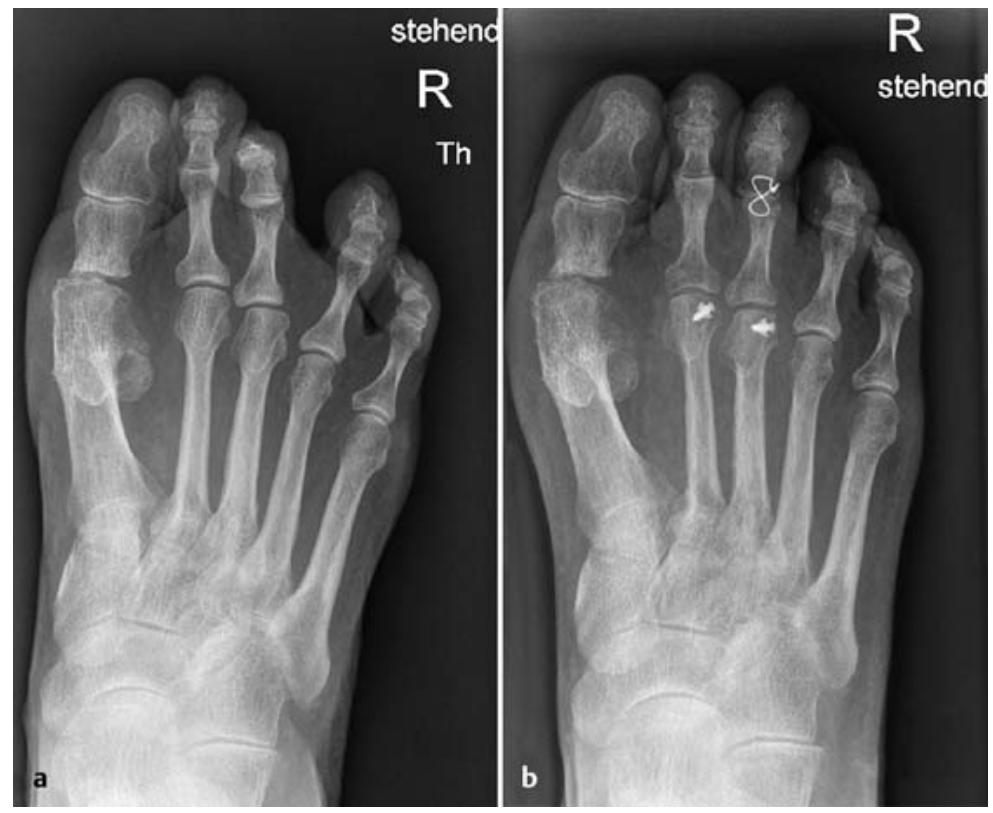

Abb. 8 - Hammerzehe: Transfermetatarsalgie II und III mit kontrakter Beugefehlstellung im PIP-III-Gelenk bei Z.n. Resektionsarthroplastik des I. Strahls. a Röntgenbilder präoperativ. b Röntgenbilder postoperativ: operative Korrektur durch eine Verkürzungsosteotomie des Metatarsale II und III mit PIP-Arthrodese der III. Zehe.

\section{Praxis}

\section{OP-Schritte und Tricks}

Tipp

Bei ausgeprägten Deformitäten und Zug der Haut am

Fußrücken kann eine K-Draht-Transfixierung hilfreich sein.

Hammerzehe. Die Hammerzehe weist eine Beugefehlstellung im PIP- und ggf. DIP-Gelenk auf. Bei flexiblen Fehlstellungen (Ausgleich im Push-up-Test) ist ein FDLSehnentransfer häufig ausreichend. Kontrakte Fehlstellungen hingegen sollten mit einer korrigierenden PIP-Gelenkarthrodese versorgt werden (Abb. 8). Den therapeutischen Algorithmus zeigt Abb. 9.

Bei älteren Patienten kann auch eine Resektionsarthroplastik des Mittelgelenks durchgeführt werden. Im Fall einer Subluxation oder Extensionsfehlstellung des Grundglieds im MTP-Gelenk können durch eine Z-förmige Verlängerung der Extensor-digitorum-longus-Sehne mit dorsaler Kapsulotomie und einem FDL-Sehnentransfer gute Korrekturergebnisse erzielt werden. Das Vorgehen ist sequenziell, d.h., dass nach jedem Schritt eine Reevaluation der Stellung und die Redressierbarkeit mittels Push-up-Test erfolgt. Bei verbleibender Deformität wird dann das nächste Verfahren durchgeführt, bis letztendlich eine vollständige Korrektur vorliegt.

- Liegt bei manuellem Ausgleich der Beugefehlstellung eine Überlänge der Zehe (länger als die medial gelegene Zehe) vor, ist zur Rezidivprophylaxe eine Verkürzungsosteotomie (Segmentresektion) der Grundphalanx in Betracht zu ziehen.

Endgelenkhammerzehe. Die Hammerzehe kann eine flexible oder kontrakte Beugefehlstellung im DIP-Gelenk aufweisen, die durch eine manuelle Redression gut differenziert werden kann. Die flexible Hammerzehe wird durch eine Tenotomie der FDL-Sehne behandelt. Bei kontrakten Fehlstellungen ist eine DIP-Gelenkarthrodese durchzuführen. Den therapeutischen Algorithmus zeigt Abb. 10.

Digitus quintus varus. Die flexible Digitus-quintusvarus-Deformität lässt sich durch einen Transfer der Extensor-digiti-minimi-Sehne i.d.R. gut korrigieren. Bei kontrakten Ausprägungen ist eine knöcherne Korrektur (Resektion der Grundgliedbasis), ggf. mit kutaner Syndaktylie, durchzuführen. 
Kleinzehenballen. Der Kleinzehenballen mit lateral prominentem Metatarsalkopf kann mit einer lateralen Kondylektomie behandelt werden. Deformitäten mit vergrößertem intermetatarsalem Winkel IV/V oder einer bogenförmigen Konfiguration des Metatarsale V können mittels distaler, diaphysärer oder basisnaher Osteotomie korrigiert werden (Einteilung s. Abb. 11).

\section{- Radiologische Befunde}

Die radiologischen Befunde sind für die Wahl des operativen Vorgehens bei Kleinzehendeformitäten von insgesamt geringer Bedeutung. Die seitliche Aufnahme lässt aufgrund der Überlagerung eine genaue Zuordnung der Zehen und Gelenke häufig nicht zu. Auf den d.-p. Aufnahmen können dorsale Subluxationen oder Luxationen im MTP-Gelenk dargestellt werden.

Die Beurteilung der Längenverhältnisse der Metatarsalia ist bei der Wahl des operativen Verfahrens hilfreich. Degenerative Veränderung und Nekrosen der Metatarsalköpfe können ebenfalls dargestellt werden und sind differenzialdiagnostisch und hinsichtlich des therapeutischen Vorgehens von Bedeutung.

\section{Operationsablauf}

Die operativen Eingriffe an den Kleinzehen können stationär oder ambulant durchgeführt werden.

\section{Lagerung}

Die operativen Verfahren zur Kleinzehenkorrektur werden in Rückenlagerung durchgeführt. Der Fuß schließt mit der unteren Tischkante ab und sollte in neutraler Stellung zu liegen kommen. Notfalls kann mithilfe von Lagerungskeilen ein Ausgleich erzielt werden. Die Operationen können in Allgemein- oder Regionalanästhesie durchgeführt werden.

\section{- Blutleere, Blutsperre}

Die Blutleere oder Blutsperre kann entsprechend dem gewählten Anästhesieverfahren am Oberschenkel (ca. 300 - $350 \mathrm{mmHg}$ ) oder am distalen Unterschenkel (200-250 mmHg) angelegt werden. Die Manschette ist mit Polsterwatte zu unterfüttern, die Blutsperre oder -leere sollte 2 Stunden nicht überschreiten.

\section{- Zugänge}

- streckseitiger longitudinaler Zugang über dem MTP-, PIP- oder DIP-Gelenk

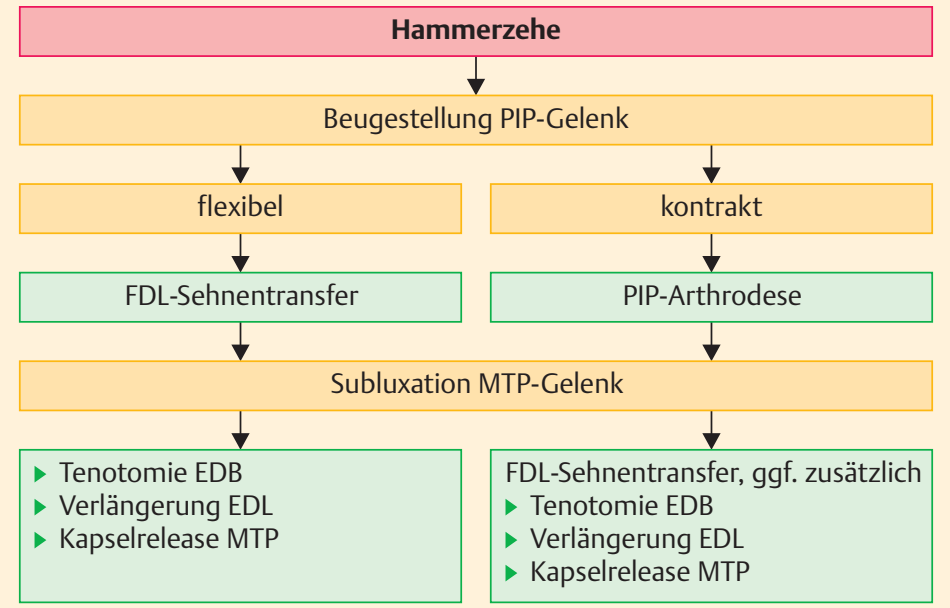

Abb. 9 - Therapeutischer Algorithmus bei der Hammerzehe. EDB = Extensor digitorum brevis; $\mathrm{EDL}=$ Extensor digitorum longus; $\mathrm{FDL}=$ Flexor digitorum longus; $\mathrm{OT}=$ Osteotomie.

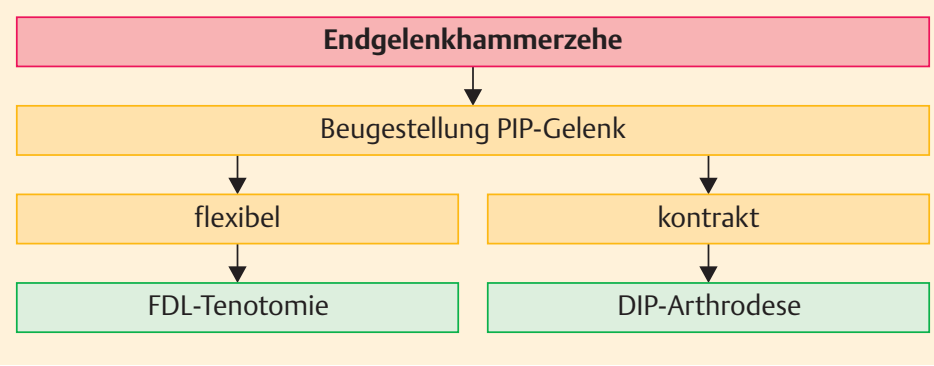

Abb. 10 - Therapeutischer Algorithmus bei der Endgelenkhammerzehe.

- streckseitiger querer Zugang über dem MTP-, PIPoder DIP-Gelenk

- plantarer querer Zugang in Höhe der Endglied- oder Grundgliedbeugefalte

- streckseitiger S-förmiger oder Z-förmiger Zugang zu den MTP-, PIP- oder DIP-Gelenken (ermöglicht den Zugang zu 2 benachbarten Grundgelenken)

\section{Operationstechnik}

- PIP-Arthrodese

- Streckseitiger longitudinaler oder querer Zugang über dem PIP-Gelenk. Der longitudinale Zugang hat den Vorteil, die Nerven- und Gefäßbahnen zu schonen und eine Erweiterung des Zugangs nach proximal oder distal zu ermöglichen. Der quere Zugang erlaubt die ovaläre Exzision eines Klavus.

- Subkutane Präparation und Darstellung der Streckersehnenhaube. Diese wird längs oder quer eröffnet und die Kollateralbänder werden durchtrennt. 


\section{Beckengürtel und untere Extremität}

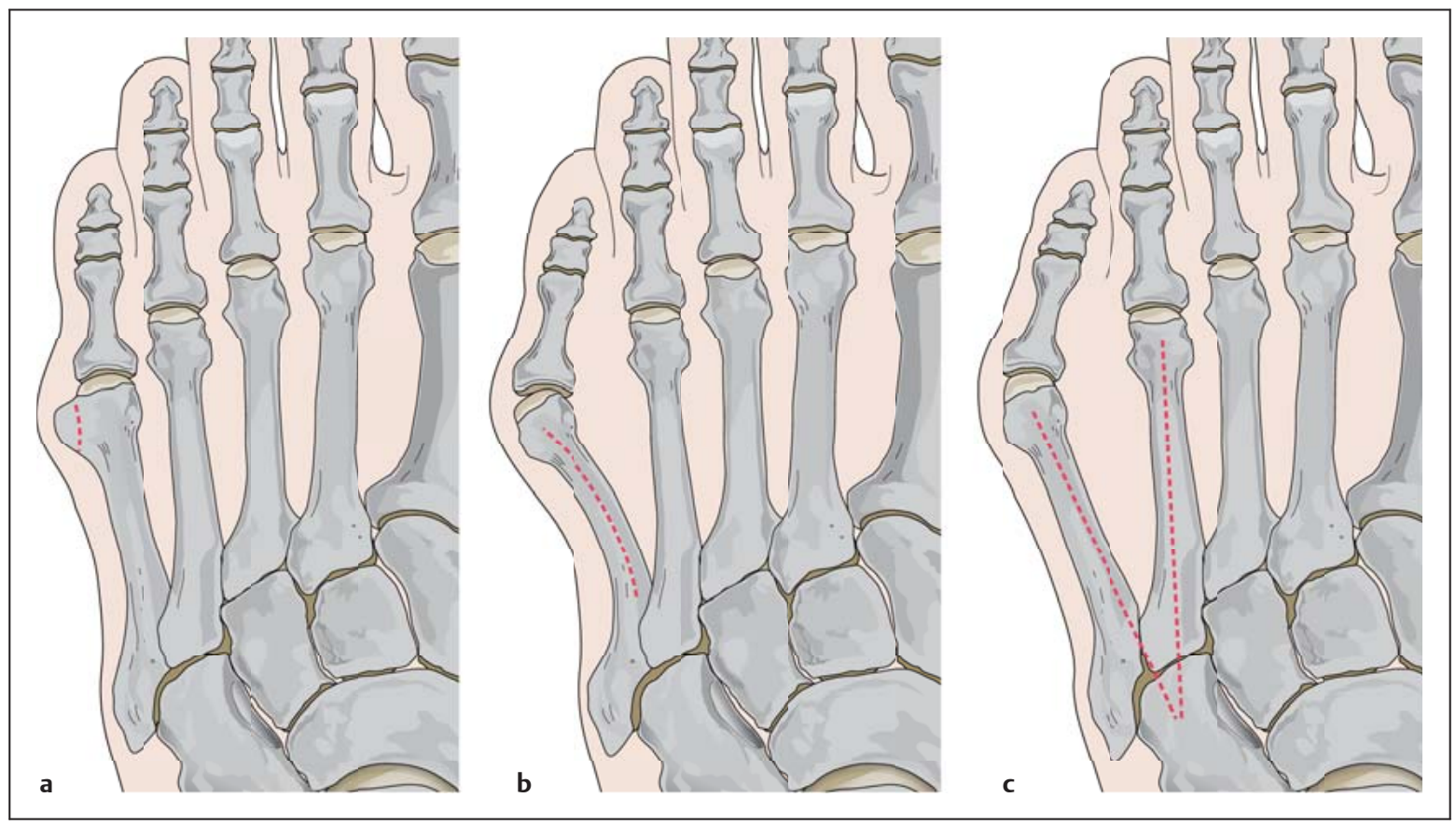

Abb. 11 - Kleinzehenballen: Einteilung. a Typ 1: Prominenter lateraler Kondylus. b Typ 2: Lateraldeviation des Metatarsale V. c Typ 3: Vergrößerter intermetatarsaler Winkel IV/V.

- Darstellung des PIP-Gelenks durch maximale Beugung.

- Sparsame Entknorpelung des Grundgliedkopfs und der Mittelgliedbasis mit der oszillierenden Säge oder dem Luer. Das Ausmaß der Präparation der Gelenkflächen ist abhängig von

- der gewählten Fixationsmethode,

- dem Grad der Fehlstellung und

- der Länge der Zehe.

- Die Fixation der Resektionsflächen kann mit einem axial geführten K-Draht (Abb. 12c), einer Drahtcerclage (Abb. 12a) oder einem intramedullären Kraftträger erfolgen (Abb. 12c). Die Zehe soll in achsgerechter Stellung korrigiert, mit Bodenkontakt der Zehenkuppe fixiert werden.

- Abschließend wird die Streckersehnenhaube readaptiert und eine Bildwandleruntersuchung oder intraoperative Röntgenkontrolle zur Überprüfung der Implantatlage und -länge, der Stellung der Zehe und der Gelenkkongruenz wird durchgeführt.

\section{- DIP-Arthrodese}

- Streckseitiger longitudinaler oder querer Zugang über dem DIP-Gelenk. Der longitudinale Zugang birgt die Gefahr einer Verletzung der Nagelbasis und kann Nagelwuchsstörungen nach sich ziehen.

- Subkutane Präparation und Darstellung der Extensordigitorum-longus-Sehne. Diese wird längs oder quer eröffnet, die Kollateralbänder werden durchtrennt.

- Darstellung des DIP-Gelenks durch maximale Beugung. Sparsame Entknorpelung mit der oszillierenden Säge oder dem Luer.

- Die Fixation der Resektionsflächen kann mit einem axial geführten K-Draht oder einer Drahtcerclage erfolgen.

- Abschließend wird die Extensor-digitorum-longusSehne readaptiert und eine Bildwandleruntersuchung oder intraoperative Röntgenkontrolle zur Überprüfung der Implantatlage und -länge, Stellung der Zehe und Gelenkkongruenz wird durchgeführt. 


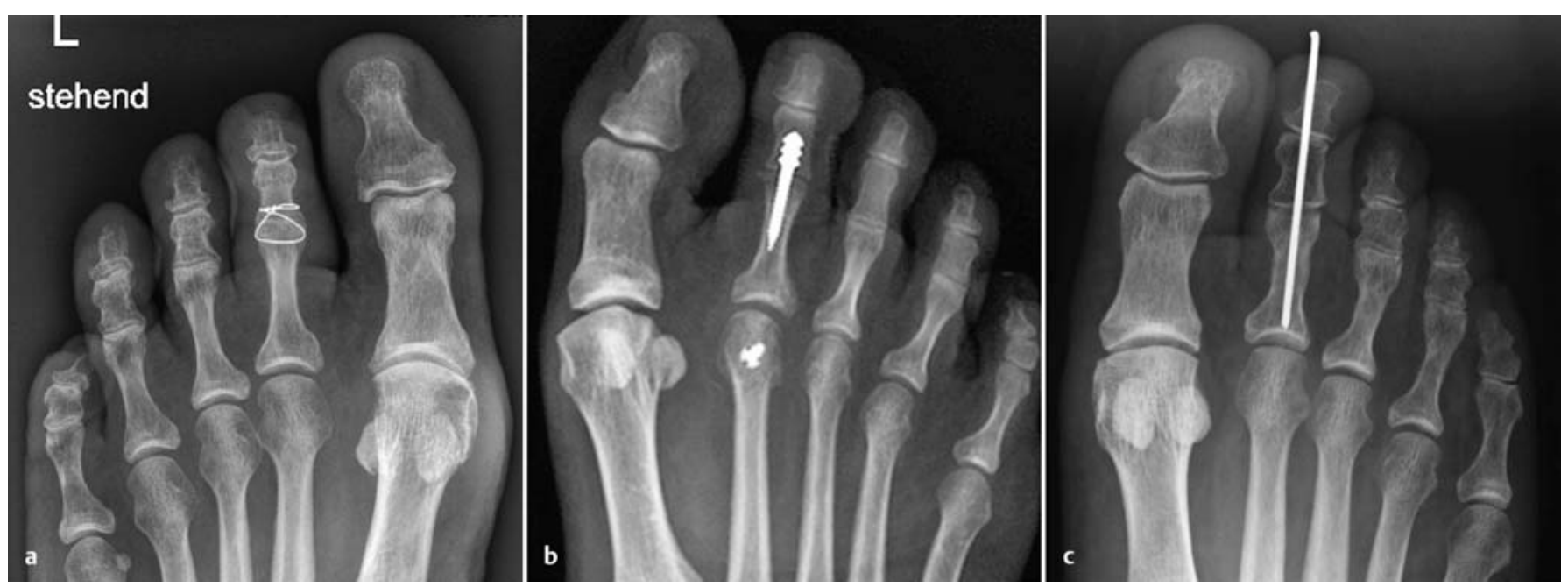

Abb. 12 - PIP-Arthrodese. a Mittels Drahtcerclage $(0,4 \mathrm{~mm})$. b Mittels intramedullärer Implantate. c Mittels K-Draht.

- Dorsale Kapsulotomie, Weichteilrelease des MTP-Gelenks und Strecksehnenverlängerung

- Streckseitiger longitudinaler Zugang über dem MTP-Gelenk.

- Subkutane Präparation bis auf die Streckeraponeurose. Z-förmige Durchtrennung der Extensor-digitorumlongus-Sehne.

- Tenotomie der lateral verlaufenden Extensor-digitorum-brevis-Sehne. Quere Inzision der Gelenkkapsel des MTP-Gelenks.

- Rekonstruktion der Streckeraponeurose unter Verlängerung der Extensor-digitorum-longus-Sehne in Neutralstellung der Zehe.

\section{Praxis}

\section{OP-Schritte und Tricks}

Tipp

Die Extensor-digitorum-brevis-Sehne kann bei einer transversalen Fehlstellung durch eine entsprechende Transposition zur Korrektur einer Valgus- oder Varusfehlstellung verwendet werden.
- Flexor-digitorum-longus-Sehnentransfer (FDL-Sehnentransfer)

- Plantare quere Inzision ein wenig proximal der Endgliedbeugefalte.

- Tenotomie der FDL-Sehnen mit dem 11er-Skalpell am Endglied. Plantare Querinzision über der Beugefalte des MTP-Gelenks.

- Subkutane Präparation durch das plantare Fettpolster bis auf die Sehnenscheide.

- Längsförmige Eröffnung der Sehnenscheide.

- Hervorluxieren der FDL-Sehne und Spaltung entlang der Raphe in 2 Sehnenzügel.

- Von plantar nach dorsal (oder wahlweise von dorsal nach plantar) mediales und laterales knochennahes Eingehen mit der gebogenen Klemme.

- Transport der beiden Sehnenzügel medial und lateral entlang der Grundphalanx nach dorsal und Ausleiten über eine dorsale Inzision.

- Die überkreuzten Sehnenzügel können in leichter Überkorrekturstellung von $10-20^{\circ}$ Plantarflexion miteinander oder mit der Extensor-digitorum-longusSehne vernäht werden.

Abb. 13 zeigt den Ablauf.

- Cave: Bei der plantaren Tenotomie oder Kapsulotomie ist ein streng medianes Vorgehen am Knochen zu wählen, um die medial und lateral verlaufenden Nerv-GefäßBahnen zu schonen. 


\section{Beckengürtel und untere Extremität}

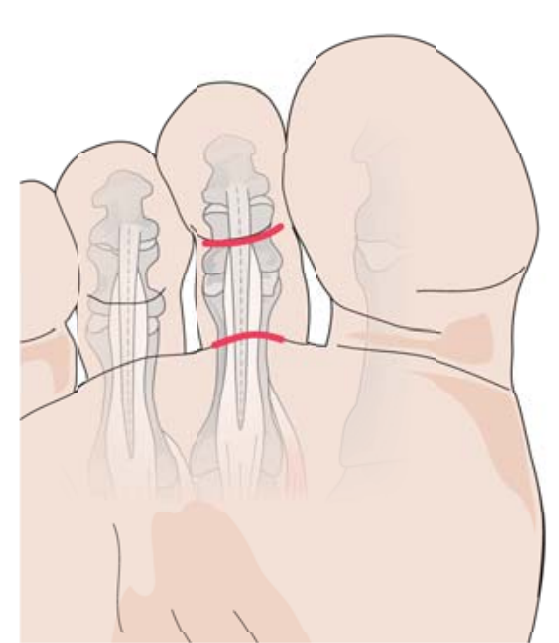

a

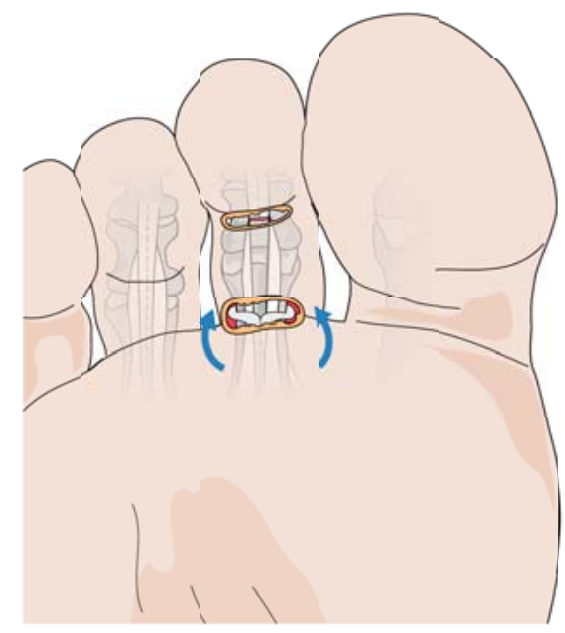

d

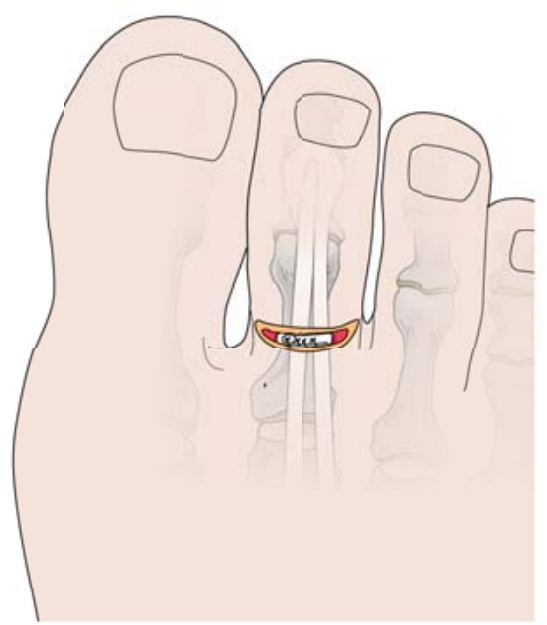

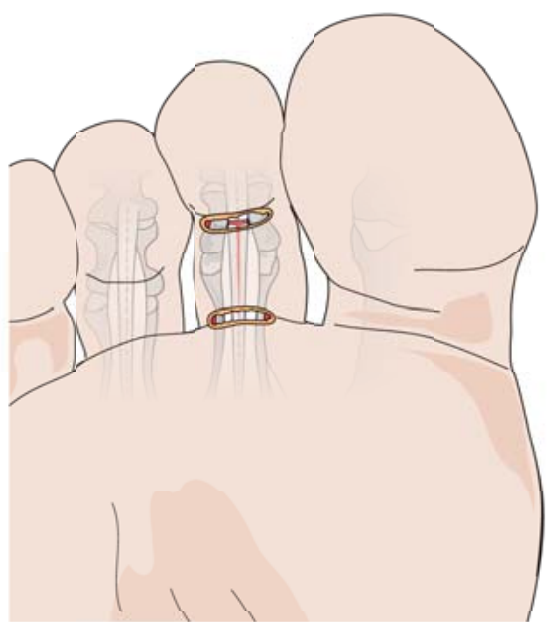

b

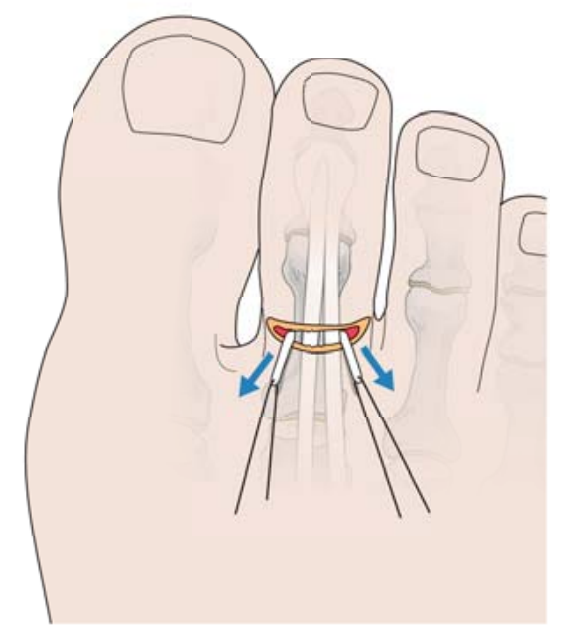

e

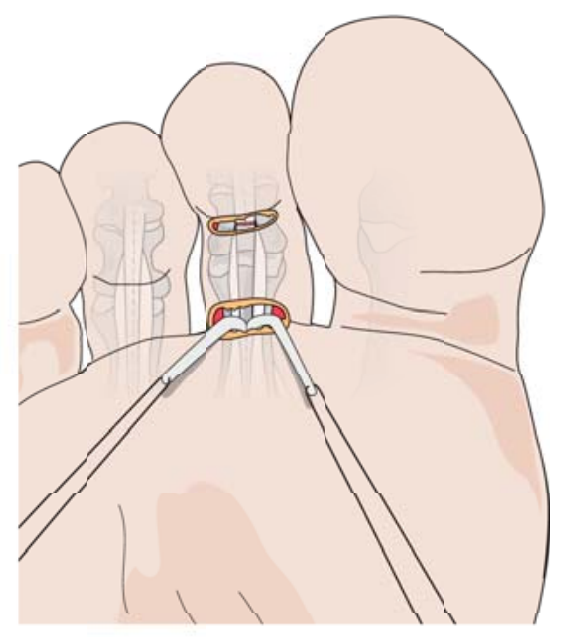

c

Abb. 13 - Flexor-digitorum-longus-Sehnentransfer: distale Ablösung der Flexor-digitorum-longus-Sehne vom Endglied. Diese wird proximal über der Beugefalte des MTP-Gelenks gespalten und knochennah nach dorsal ausgeleitet. Die überkreuzten Sehnenzügel werden in Korrekturstellung der Zehe miteinander vernäht.

a Plantare quere Inzision ein wenig proximal der Endgliedbeugefalte. b Distale Tenotomie über eine Stichinzision. c Hervorluxieren der Flexor-digitorum-longus-Sehne und Spaltung entlang der Mittellinie. d Knochennahe Präparation und Transfer der Sehnenzügel nach dorsal. e Ausleiten der beiden Sehnenzügel über eine dorsale Inzision. $\mathbf{f}$ Vernähen der beiden überkreuzten Sehnenzügel. $\mathbf{g}$ Hautnaht. 
Praxis

\section{OP-Schritte und Tricks}

Tipps

Durch entsprechenden Zug an den dorsal überkreuzten

Sehnenzügeln lassen sich transversale Fehlstellungen korrigieren. Optional kann zusätzlich ein mediales oder laterales Kapselrelease durchgeführt werden. Nach der longitudinalen Eröffnung der Sehnenscheide sind 3 Sehnen zu erkennen. Die FDL-Sehne liegt tiefer und zentral. Sie weist im Gegensatz zur Flexor-digitorumbrevis-Sehne eine zentrale Raphe auf.

\section{- Metatarsale Verkürzungsosteotomie} (Weil-Osteotomie)

- Streckseitiger longitudinaler Zugang über dem MTP-Gelenk.

- Subkutane Präparation bis auf die Streckeraponeurose. Retraktion oder Z-förmige Durchtrennung der Extensor-digitorum-longus-Sehne.

- Optionale Tenotomie der lateral verlaufenden Extensor-digitorum-brevis-Sehne.

- Distales Release der Kollateralbänder an der Grundphalanx.

- Maximale Plantarflexion der Zehe im MTP-Gelenk.

- Langstreckige schräge Osteotomie des Metatarsale beginnend am proximalen Ende des metatarsalen Knorpels, ca. 1-2 mm unterhalb der dorsalen Knorpelbegrenzung (Abb. 14b).

- Verschiebung des Metatarsalkopfs nach proximal entsprechend der präoperativen Planung um 3-10 mm (Abb. 14c).

- Osteosynthese mit einer 2,0- bis 2,5-mm-Schraube der Länge 10-12 mm von dorsal in den Metatarsal-

\section{Praxis}

\section{OP-Schritte und Tricks}

\section{Tipps}

Die Osteotomie soll parallel zur Belastungsebene des Fußes durchgeführt werden. Sie ist entscheidend für die Verschiebung in der Sagittalebene. Erfolgt sie zu steil, d. h. zu sehr nach plantar abfallend, wird mit der Proximalisierung auch eine Plantarisierung des Kopfes erzielt. Diese kann zu einer erhöhten Druckbelastung des Metatarsalkopfs mit Ausbildung oder Persistenz der Metatarsalgie und der Hyperkeratosen führen. kopf. Abtragen des überstehenden dorsalen Knochens mit dem Luer (Abb. 14d).

\section{- Transfer der Extensor-digiti-quinti-Sehne} auf den Ansatz des M. abductor digiti quinti (Sehnentransfer nach Lapidus)

- S-förmige Inzision von der Zehenzwischenfalte IV/V nach lateral-proximal verlaufend über das dorsale an das laterale MTP-V-Gelenk.

- Subkutane Präparation und Darstellung der Extensordigiti-quinti-Sehne. Diese wird mit einer Klemme unterfahren, angespannt und im Verlauf auf dem Fußrücken identifiziert.

- Etwa $5 \mathrm{~cm}$ proximal des MTP-Gelenks erfolgt eine Inzision über der Sehne, die mit einer Klemme hervorluxiert, quer tenotomiert und von distal herausgezogen wird (Abb. 15a).

- Präparation des MTP-Gelenks mit Release der verkürzten medialen (Gelenkkapsel, Kollateralbänder) und dorsoplantaren Strukturen.

- Präparation der Extensor-digiti-quinti-Sehne bis zur distalen Grundphalanx. Die Sehne wird dann von medial nach plantar unter der Grundphalanx nach lateral-proximal ausgeleitet (Abb. 15b).

- Das ausgeleitete freie Sehnenende wird unter entsprechender Vorspannung in leichter Überkorrekturstellung der Zehe auf den M. abductor minimi genäht.

- Optional kann die Sehne auch durch einen transossären Kanal ausgeleitet werden.

\section{Praxis}

\section{OP-Schritte und Tricks}

\section{Tipp}

Das distale Hervorluxieren der Sehne kann durch Querzügel erschwert sein. Die Sehne kann in diesen Fällen mit einer geraden Klemme oder einem flexiblen Sehnenstripper gelöst und mühelos geborgen werden.

\section{n Naht der plantaren Platte}

Der Zugang, die Präparation und Osteotomie erfolgen wie bei der metatarsalen Verkürzungsosteotomie (s.o).

- Der Metatarsalkopf wird mindestens $10 \mathrm{~mm}$ nach proximal verschoben und mit einem K-Draht transfixiert.

- Das MTP-Gelenk wird distrahiert und die plantare Platte aufgesucht. 


\section{Beckengürtel und untere Extremität}

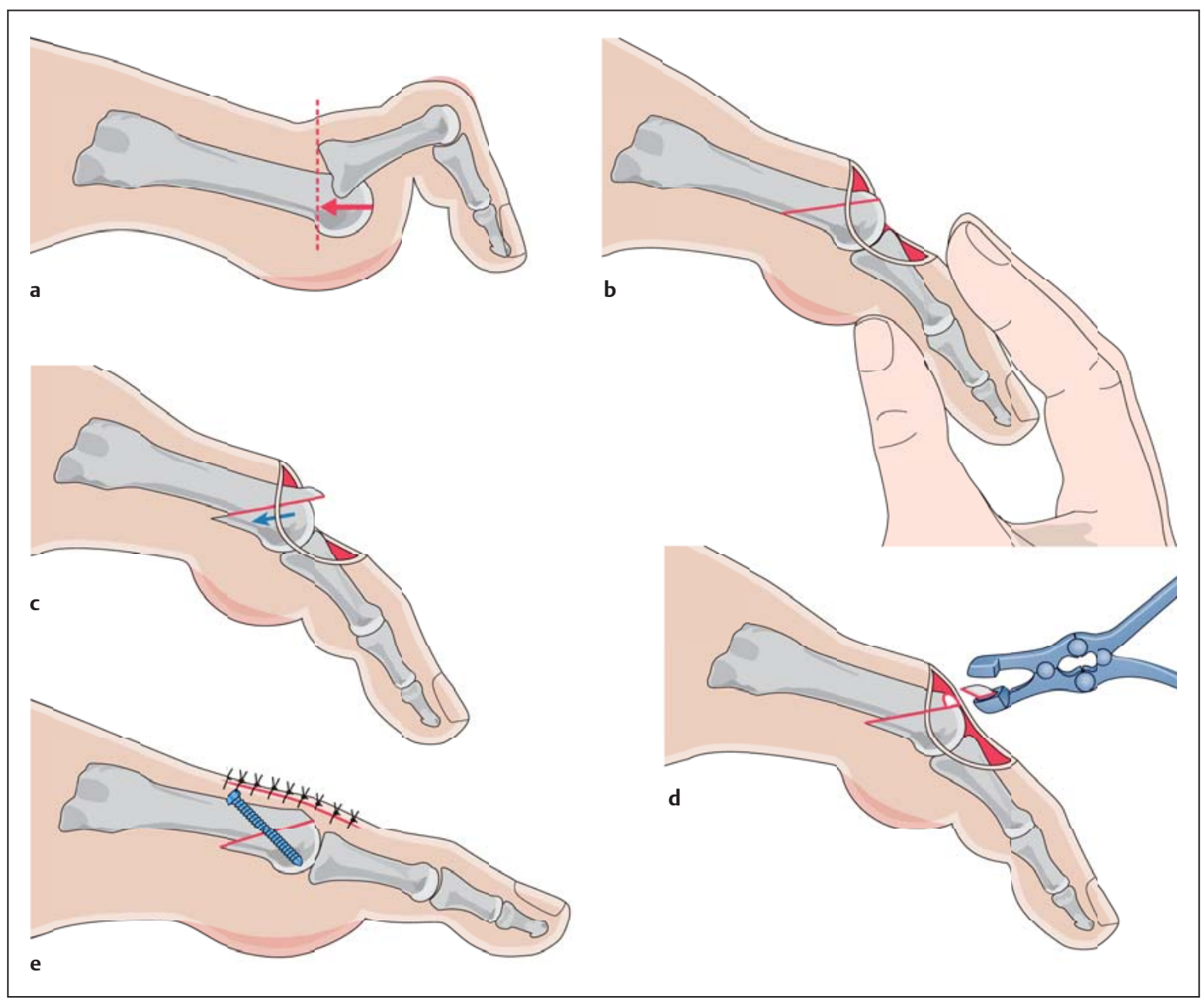

Abb. 14 - Metatarsale Verkürzungsosteotomie nach Weil. a Subluxation des MTP-Gelenks. b Langstreckige Osteotomie des Metatarsale parallel zur Belastungsebene. c Verschiebung des Metatarsalkopfs nach proximal um 3-10 mm. d Abtragen des überstehenden Knochens. e Osteosynthese in geplanter Proximalisierung.

- Longitudinale Rupturen können Seit-zu-Seit genäht werden. Bei transversalen Partialrupturen der plantaren Platte empfiehlt sich eine vollständige distale Ablösung, um die Instrumentierung zu erleichtern.

- Anrauen der Insertionsstelle an der plantaren Grundphalanx.

- Anschlingen des distalen Endes der plantaren Platte mithilfe einer kleinen Nadel oder Fasszange bzw. eines Fadendurchziehers.

- Im Bereich der proximalen Phalanx werden 2 senkrechte oder quere Bohrlöcher von dorsal nach plantar angelegt. Durch diese werden die Fadenenden der angeschlungenen plantaren Platte nach dorsal gezogen.

- Fixierung des Metatarsalkopfs in der gewünschten Position.

- Raffung der plantaren Platte durch Zug und Verknoten der Fadenenden in leichter Plantarflexionsstellung der Zehe.
- Alternativ ist eine Naht der plantaren Platte von plantar möglich.

\section{- Phalangeale Closing-Wedge-Osteotomie}

- Streckseitiger longitudinaler Zugang über der proximalen Grundphalanx.

- Subkutane Präparation und Darstellung der Extensordigitorum-longus-Sehne. Diese wird retrahiert und die proximale Grundphalanx wird dargestellt.

- Basisnahe mediale oder laterale Keilosteotomie der Grundphalanx unter Erhalt der Gegenkortikalis.

- Schließen der Osteotomie und Fixation mit K-Drähten oder einer Cerclage. 


\section{Kleinzehenfehlstellungen}

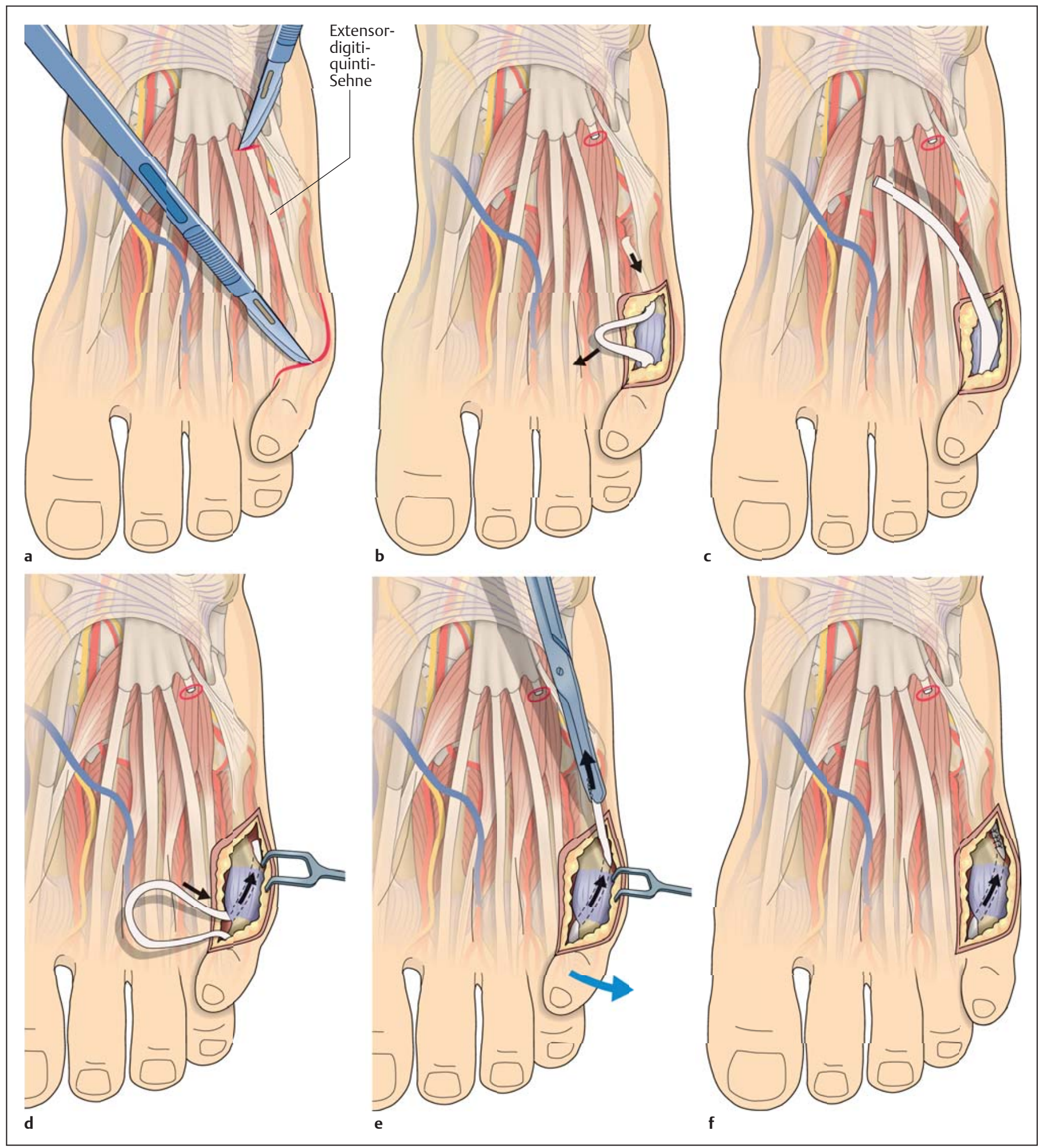

Abb. 15 - Transfer der Extensor-digiti-quinti-Sehne auf den Ansatz des M. abductor digiti quinti (Sehnentransfer nach Lapidus). a S-förmige Inzision bis in die Beugefalte zwischen DIV und DV. b Proximale Tenotomie der Extensor-digiti-quinti-Sehne. c Ausleiten der Sehne nach distal. d Die Extensor-digiti-quinti-Sehne wird unter der Grundphalanx nach lateral ausgeleitet. e Durch Zug an der Sehne kann die Zehe in die gewünschte Stellung gebracht werden. $\mathbf{f}$ Die Extensor-digiti-quinti-Sehne wird auf den M. abductor minimi fixiert. 
- Kapselraffung MTP-Gelenk

- Streckseitiger longitudinaler Zugang über dem MTP-Gelenk.

- Subkutane Präparation und Darstellung der Extensordigitorum-longus-Sehne. Diese wird Z-förmig verlängert oder retrahiert. Dorsales und entsprechend der Fehlstellung mediales oder laterales MTP-Kapselrelease. Bei der häufigeren Medialdeviation der Zehe erfolgen das Release medial an der Basis der Grundgliedphalanx und die Raffung lateral in Korrekturstellung der Zehe.

\section{- Transfer der Extensor-digitorum-brevis-Sehne}

- Streckseitiger longitudinaler oder S-förmiger Zugang über dem MTP-Gelenk.

- Subkutane Präparation und Darstellung der Extensordigitorum-longus- und -brevis-Sehnen.

- Eröffnung der Strecksehnenaponeurose und dorsomediales Kapselrelease sowie Durchtrennung der medialen Kollateralbänder bei entsprechender varischer Fehlstellung der Zehe.

- Die lateral verlaufende Extensor-digitorum-brevisSehne wird distal tenotomiert und armiert.

- Von distal Unterfahren des Lig. intermetatarsale transversum und subligamentäres Durchziehen der Extensor-digitorum-brevis-Sehne von proximal nach distal.

- Fixation des Sehnenstumpfs in geringer Überkorrektur über eine transossäre Naht oder einen Knochenanker am Grundglied.

\section{- Laterale Kondylektomie und distale Chevron- Osteotomie des Metatarsale V (Chevronette)}

- Lateralseitiger longitudinaler Zugang zum MTP-VGelenk.

- Subkutane Präparation und Darstellung der Gelenkkapsel.

- Longitudinale oder L-förmige Inzision der Gelenkkapsel und Darstellung der lateralen Kondyle. Abhängig von der Ausprägung der lateralen Kondyle Abtragen mit der oszillierenden Säge von distal nach proximal in Verlängerung zur Schaftachse.

- Einbohren eines K-Drahts von lateral in das Zentrum des Metatarsalkopfs zur Festlegung der Osteotomieebene.

- Durchführen einer V-förmigen Osteotomie in einem Winkel von ca. $60^{\circ}$.

- Manuelle Verschiebung des Metatarsalkopfs nach medial um 30-50\% der Metaphysenschaftbreite.
Praxis

\section{OP-Schritte und Tricks}

Tipp

Der gewissenhafte Verschluss der Gelenkkapsel soll die Rezidivwahrscheinlichkeit reduzieren. Bei einer L-förmigen Inzision kann die Kapsel durch transossäre oder transperiostale Fixation gut gerafft und sicher verschlossen werden.

- Fixierung des Kopfes mit einem K-Draht oder wahlweise einer Schraubenosteosynthese.

- Abtragen des überstehenden lateralen Knochens mit der oszillierenden Säge.

- Sorgfältige Kapselnaht.

\section{- Diaphysäre Schrägosteotomie Metatarsale V}

- Lateralseitiger longitudinaler Zugang zum MTP-VGelenk und Metatarsalschaft.

- Subkutane Präparation und Darstellung der Gelenkkapsel.

- Longitudinale oder L-förmige Inzision der Gelenkkapsel und Darstellung der lateralen Kondyle. Abhängig von der Ausprägung der lateralen Kondyle Abtragen mit der oszillierenden Säge von distal nach proximal in Verlängerung zur Schaftachse.

- Präparation des Metatarsalschafts nach proximal.

- Retraktion des M. abductor minimi nach plantar.

- Darstellen der Metatarsaldiaphyse und horizontale Osteotomie mit der oszillierenden Säge von dorsoproximal nach plantar-distal.

- Verschieben des dorsalen Fragments nach medial, bis die gewünschte Korrektur erzielt wurde.

- Fixation mit 2 Schrauben oder wahlweise 2 Drahtcerclagen.

- Abtragen des überstehenden Knochens.

Tab. 1 fasst das operative Vorgehen bei den verschiedenen Kleinzehenfehlstellungen zusammen.

\section{Komplikationen}

Früh- und Spätkomplikationen können trotz sorgfältiger Planung und Durchführung der operativen Korrektur von Kleinzehenfehlstellungen auftreten. Insgesamt ist die Komplikationsrate jedoch gering. 


\section{Frühkomplikationen}

Postoperative Schwellung. Sie ist durch unmittelbare und konsequente Hochlagerung, Kühlung und entsprechende Schonung des Fußes sowie Lymphdrainagen zu behandeln.

Sensibilitätsstörungen. Verletzungen oder Irritation der Nn. digitales dorsales proprii sind häufig innerhalb von 6 Monaten regrediert. Eine durch eine Schwellung hervorgerufene Sensibilitätsstörung verbessert sich i.d.R. mit Abschwellen der Zehe.

Infektionen. Infektionen erfordern eine engmaschige Überwachung mit gezielter systemischer Antibiotikagabe nach Resistenzbestimmung. Bei ausgeprägten Infekten sind ein operatives Débridement und die Entfernung der Implantate durchzuführen.

Wundheilungsstörungen. Je nach Ausprägung ist eine frühzeitige chirurgische Revision erforderlich.

Postoperative Ischämie. Nach aufwendigen Achskorrekturen kann es zu meist temporären Minderdurchblutungen der betroffenen Zehen kommen. Begünstigt werden diese durch eine Dehnung der Gefäße (Achskorrektur) oder durch kombinierte plantare und dorsale Zugänge mit Verletzung der entsprechenden Gefäße. Anhaltende Minderdurchblutungen können mit einer irreversiblen Nekrose der Zehe einhergehen.

\section{- Spätkomplikationen}

Über- und Unterkorrektur. Bei sehr ausgeprägten Fehlstellungen ist mit dem Patienten präoperativ eine realistische Erwartungshaltung hinsichtlich der Korrekturmöglichkeiten zu klären. Unterkorrekturen sind insgesamt häufiger als Überkorrekturen. Durch redressierende Maßnahmen kann versucht werden, flexible Fehlstellungen zu einem gewissen Maß zu korrigieren. Bei ausgeprägten Über- oder Unterkorrekturen sollte mit dem Patienten über eine weitere operative Maßnahme gesprochen werden. Die metatarsale Verkürzungsosteotomie nach Weil kann bei starker Proximalisierung des Metatarsalkopfs zu einer instabilen Zehe („floppy toe“) führen.

Rezidiv. Bei Rezidiven sollte eine erneute Analyse der Fehlstellung erfolgen. Im Fall einer flexiblen Fehlstellung kann ein zusätzlicher weichteiliger Eingriff (Sehneneingriff oder Release des MTP-Gelenks) zur Korrektur führen. Im Fall von kontrakten Fehlstellungen ist ggf. ein erneuter knöcherner Eingriff erforderlich. Ähnlich wie bei
Tabelle 1

Vorgehen bei Kleinzehenfehlstellungen.

\begin{tabular}{|c|c|c|}
\hline Fehlstellung & & empfohlene Maßnahmen \\
\hline \multirow[t]{2}{*}{$\begin{array}{l}\text { Krallen- oder } \\
\text { Klauenzehe }\end{array}$} & MTP flexibel & $\begin{array}{l}\text { FDL-Sehnentransfer, } \\
\text { dorsales Weichteilrelease }\end{array}$ \\
\hline & MTP kontrakt & $\begin{array}{l}\text { dorsales Weichteilrelease + } \\
\text { metatarsale Verkürzungsosteotomie, } \\
\text { ggf. FDL-Sehnentransfer, } \\
\text { ggf. PIP-Arthrodese }\end{array}$ \\
\hline
\end{tabular}

\begin{tabular}{|c|c|c|}
\hline $\begin{array}{l}\text { Crossover-Toe- } \\
\text { Deformität }\end{array}$ & & $\begin{array}{l}\text { wie kontrakte Klauenzehe } \\
\text { und Naht der plantaren Platte }\end{array}$ \\
\hline \multirow[t]{3}{*}{ Hammerzehe } & $\begin{array}{l}\text { flexibel } \\
\text { (Zehenlänge } 1=2 \text { ) }\end{array}$ & FDL-Sehnentransfer \\
\hline & $\begin{array}{l}\text { flexibel } \\
\text { (Zehenlänge } 1<2 \text { ) }\end{array}$ & $\begin{array}{l}\text { FDL-Sehnentransfer, } \\
\text { ggf. Segmentresektion } \\
\text { Grundgliedphalanx }\end{array}$ \\
\hline & kontrakt & $\begin{array}{l}\text { PIP-Gelenkarthrodese, } \\
\text { ggf. FDL-Sehnentransfer }\end{array}$ \\
\hline \multirow{2}{*}{$\begin{array}{l}\text { Endgelenk- } \\
\text { hammerzehe }\end{array}$} & flexibel & Tenotomie FDL-Sehne \\
\hline & kontrakt & DIP-Gelenkarthrodese \\
\hline \multirow{2}{*}{$\begin{array}{l}\text { Digitus quintus } \\
\text { varus }\end{array}$} & flexibel & Transfer Extensor-digiti-quinti-Sehne \\
\hline & kontrakt & $\begin{array}{l}\text { Resektion der Grundgliedbasis, } \\
\text { ggf. kutane Syndaktylie }\end{array}$ \\
\hline \multirow[t]{3}{*}{ Kleinzehenballen } & $\begin{array}{l}\text { lateral prominenter } \\
\text { Metatarsalkopf }\end{array}$ & $\begin{array}{l}\text { laterale Kondylektomie } \\
\text { und ggf. distale Osteotomie }\end{array}$ \\
\hline & $\begin{array}{l}\text { bogenförmige } \\
\text { Konfiguration } \\
\text { Metatarsale V }\end{array}$ & $\begin{array}{l}\text { diaphysäre Osteotomie } \\
\text { und ggf. laterale Kondylektomie }\end{array}$ \\
\hline & $\begin{array}{l}\text { vergrößerter } \\
\text { Intermetatarsalwinkel } \\
\text { (IM-Winkel) IV } / \mathrm{V}>10^{\circ}\end{array}$ & $\begin{array}{l}\text { distale, diaphysäre oder basisnahe } \\
\text { Osteotomie } \\
\text { und ggf. laterale Kondylektomie }\end{array}$ \\
\hline
\end{tabular}

den Über- und Unterkorrekturen sollte diese Entscheidung individuell erfolgen.

Pseudarthrose. Pseudarthrosen nach PIP- oder DIPArthrodese sind nicht selten, führen aber i.d.R. zu einer fibrotischen Stabilisierung ohne klinische Relevanz. Bei Beschwerden ist eine operative Revision mit Rearthrodese und ggf. autogenem Knochenmaterial zu erwägen. 
Materialkonflikt. Durch die eingebrachten Implantate (Schraube, Cerclagen, K-Drähte) kann es zu einer Irritation des Weichteilgewebes kommen. Diese Materialien können nach knöcherner Konsolidierung entfernt werden.

\section{Nachbehandlung}

Der Nachbehandlung operativer Korrekturen von Kleinzehenfehlstellungen kommt eine besondere Bedeutung $\mathrm{zu}$.

Ruhigstellung. Durch die Anlage eines intraoperativen redressierenden Verbands werden die Zehen in entsprechender Stellung im MTP-, PIP- und DIP-Gelenk stabilisiert (Abb. 16). Dieses Vorgehen ermöglicht eine Heilung der weichteiligen und knöchernen Strukturen in der gewünschten Position. Die redressierenden Verbände sollten für mindestens 3 Wochen postoperativ angelegt werden. Anschließend kommen für bis zu 12 Wochen nach der Operation konfektionierte Bandagen oder Tapeverbände zum Einsatz.

Funktionelle Beübung. Aufgrund der stabilen Versorgung der Osteotomien ist eine funktionelle Nachbehandlung des operierten Fußes mit Vollbelastung in einer Orthese mit starrer Sohle möglich. Unterarmgehstützen sind nicht zwingend erforderlich, können jedoch bei anfänglichen Schmerzen und Gangunsicherheit von Nutzen sein.

Röntgenkontrollen. Postoperativ sollte eine Röntgenkontrolle des operierten Fußes im Stand in 2 Ebenen (d.-p. und streng seitlich) erfolgen. Nach 6 Wochen ist
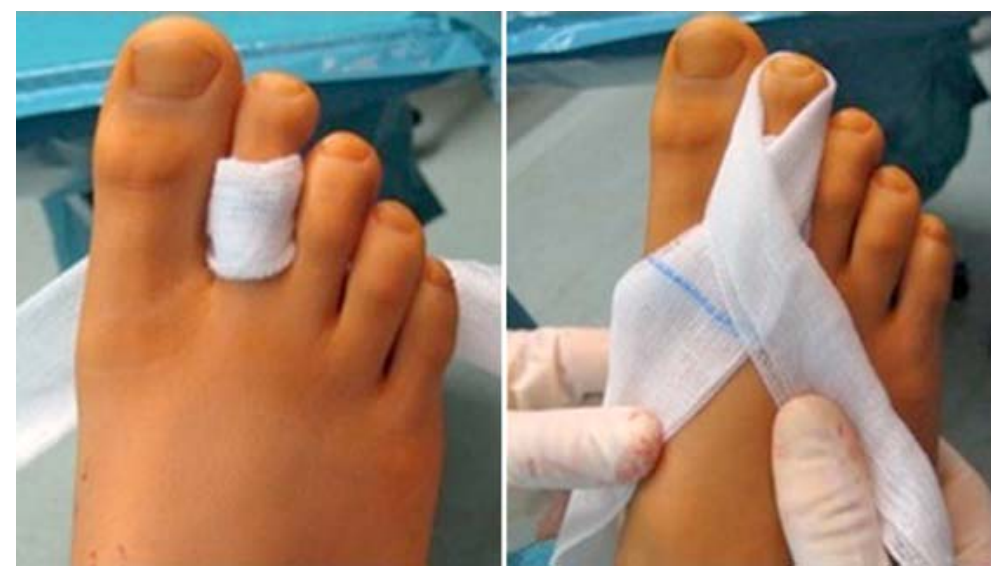

Abb. 16 - Redressierende Verbandanlage: lange, ausgezogene Kompressen, die in Achtertouren um das Grund- und Mittel-/Endglied gelegt werden. a Die proximale Kompresse redressiert das Grundglied nach plantar. b Die distale Kompresse redressiert das Mittel- und Endglied nach dorsal. eine abschließende Röntgenkontrolle sinnvoll, um die knöcherne Heilung beurteilen zu können.

Schuhwerk. Nach knöcherner Konsolidierung ist der Wechsel in konfektioniertes Schuhwerk möglich. Dabei sollte auf eine entsprechende Passform geachtet werden. Eine Einlagenversorgung ist nicht zwingend, kann aber 12 Wochen postoperativ erfolgen.

Materialentfernung. Eine Materialentfernung ist i.d.R. nicht erforderlich und nur bei störendem Material indiziert.

Arbeitsunfähigkeit. Die Dauer beträgt, abhängig vom Ausmaß der operativen Korrektur, der Anzahl der operierten Zehen und der beruflichen Tätigkeit 6-8 Wochen.

\section{Perspektiven}

Die differenzierte Analyse von Kleinzehenfehlstellungen unter Berücksichtigung der Pathophysiologie sowie der sequenzielle Einsatz weichteiliger und knöcherner Korrekturverfahren führt zu guten postoperativen Ergebnissen. Seit wenigen Jahren erfreut sich im deutschsprachigen Raum die perkutane oder minimalinvasive Fußchirurgie größerer Beliebtheit. Diese wird als Minimal Incision Surgery (MIS) bereits seit längerer Zeit in den USA, Frankreich und in den südeuropäischen Ländern praktiziert. Die Prinzipien der MIS-Fußchirurgie unterscheiden sich teilweise wesentlich von denen im Artikel dargestellten offenen Vorgehensweisen.

Neben weichteilreleasenden Verfahren werden v.a. minimalinvasive Osteotomien an den Zehen und den Metatarsalia durchgeführt. Die Operationstechniken benötigen keine ausgedehnten Zugänge, sondern erfolgen über definierte Stichinzisionen. Die Osteotomien werden mithilfe motorbetriebener Fräsen und Bohrer perkutan durchgeführt. Bei der Nachbehandlung kommt der stabilisierenden und redressierenden Verbandanlage eine noch größere Bedeutung zu als bei den offenen Techniken.

Die Ergebnisse der MIS-Fußchirurgie an den Kleinzehen scheinen erfolgversprechend, auch wenn belastbare wissenschaftliche Arbeiten zurzeit nicht vorliegen. Inwieweit diese MIS-Techniken die offenen Operationsverfahren ergänzen oder gar ablösen werden, bleibt abzuwarten. Die MIS-Chirurgie muss sich dafür mit den guten Ergebnissen der offenen Kleinzehenchirurgie messen lassen. 


\section{Zusammenfassung}

Fehlstellungen der Kleinzehen können isoliert, häufiger aber im Rahmen einer Spreizfußdeformität und HV-Fehlstellung auftreten. Neben einer alters- und geschlechtsgebundenen Häufung scheinen auch externe Faktoren (Schuhwerk) eine Entstehung zu begünstigen.

Die Störung der Balance aktiv und passiv stabilisierender Strukturen des Metatarsophalangealgelenks führt zu einer Veränderung der Biomechanik und letztendlich zu der Ausbildung unterschiedlicher Kleinzehenfehlstellungen. Die geläufigen Begriffe Klauen-, Hammer- und Krallenzehe bezeichnen unterschiedliche Fehlstellungen der entsprechenden Gelenke, sie sind jedoch in der medizinischen Terminologie nicht einheitlich definiert.

Für den Patienten kann in Abhängigkeit von der Ausprägung der Fehlstellung eine deutliche Einschränkung der Lebensqualität und Aktivität bestehen. Die konservative Therapie zielt auf eine Linderung der Beschwerden, ohne wesentlich in die Pathomechanik einzugreifen. Die Auswahl des Schuhwerks mit ausreichender Vorfußbreite und Höhe, die Einlagenversorgung als auch orthopädieschuhtechnische Maßnahmen sind Pfeiler der konservativen Therapie. Diese können durch fußpflegerische und physiotherapeutische Behandlungen sowie eine Orthesenversorgung ergänzt werden.

Die konservativen Maßnahmen sind bei ausgeprägten Fehlstellungen häufig nicht ausreichend, sodass in diesen Fällen die Indikation zur Operation geprüft werden sollte. Die operative Therapie zielt auf eine Korrektur der Fehlstellungen und Schaffung physiologischer Vorfußverhältnisse hin. Sie soll eine Schmerzlinderung und eine Verbesserung der Funktion des Fußes erzielen. Die Wahl der operativen Vorgehensweise wird vom klinischen Befund und der dezidierten Analyse der Fehlstellungen im Verständnis der komplexen Pathomechanik bestimmt. Algorithmen helfen bei der Therapieentscheidung; patientenrelevante Faktoren wie das Alter, der Bewegungsanspruch und Begleiterkrankungen (Diabetes mellitus, rheumatoide Arthritis) müssen dabei aber Beachtung finden.

Postoperativ ist eine frühfunktionelle Behandlung mit Vollbelastung in einer sohlensteifen Orthese möglich. Der redressierenden Verbandstechnik kommt zur Sicherung des Korrekturergebnisses eine besondere Bedeutung zu.
Interessenkonflikt: Die Autoren bestätigen, dass kein Interessenkonflikt vorliegt.

\section{Zum Weiterlesen und Vertiefen}

Coughlin MJ. Crossover second toe deformity. Foot Ankle 1987; 8: 29-39

Coughlin MJ. Lesser Toe Deformities. In: Coughlin MJ, Saltzman CL, Anderson RB, eds. Mann's Surgery of the Foot and Ankle. 9th ed. Philadelphia: Elsevier Saunders; 2014: 322-424

Coughlin MJ, Dorris J, Polk E. Operative repair of the fixed hammertoe deformity. Foot Ankle 2000; 21: 94-104

Dohle J. Kleinzehenfehlstellungen. In: Rammelt S, Hrsg. Fuß- und Sprunggelenkschirurgie. Das Kursbuch. Stuttgart: Schattauer; 2014: $137-153$

Gregg J, Silberstein M, Clark C et al. Plantar plate repair and Weil osteotomy for metatarsophalangeal joint instability. J Foot Ankle Surg 2007; 13: 116-121

Harris W, Mote GA, Malay DS. Fixation of the proximal interphalangeal arthrodesis with the use of an intraosseous loop of stainless-steel wire suture. J Foot Ankle Surg 2009; 48: 411 - 414

Hofstaetter SG, Hofstaetter JG, Petroutsas JA et al. The Weil osteotomy: A seven year follow-up. J Bone Joint Surg $\mathrm{Br} 2005$; 87: 1507 - 1511

Myerson NS, Shereff NJ. The pathological anatomy of claw and hammer toes. J Bone Joint Surg Am 1989; 71: 45-49

Scheck M. Etiology of acquired hammer toe deformity. Clin Orthop 2009; 123: $63-67$

Thompson FM, Deland JT. Flexor tendon transfer for metatarsophalangeal instability of the second toe. Foot Ankle 1993; 14: 385-388

\section{Korrespondenzadresse}

Dr. med. Dariusch Arbab

Orthopädische Klinik

Mitglied der Fakultät Gesundheit Universität Witten/Herdecke Klinikum Dortmund

Beurhausstraße 40

44137 Dortmund

Telefon: + $49(0) 231953-21850$

Fax: $\quad+49(0) 231953-21019$

E-Mail: darbab@gmx.de 


\section{CME-Fragen}

Wodurch ist die Epidemiologie von Kleinzehenfehlstellungen gekennzeichnet?

\section{CME.thieme.de}

\section{CME-Teilnahme}

- Viel Erfolg bei Ihrer CME-Teilnahme unter http://cme.thieme.de

- Bitte informieren Sie sich vorab online über die Gültigkeitsdauer.

- Sollten Sie Fragen zur Online-Teilnahme haben, unter http://cme.thieme.de/hilfe finden Sie eine ausführliche Anleitung.
Trochanter major

A Kleinzehenfehlstellungen sind i.d.R. angeboren und bereits im Kindesalter auffällig.

B Kleinzehenfehlstellungen machen einen großen Anteil der Operationen am Vorfuß aus.

C Kleinzehenfehlstellungen betreffen Frauen und Männer in gleichen Anteilen.

D Kleinzehenfehlstellungen haben zwischen dem 40. und 50. Lebensjahr ihren Häufigkeitsgipfel.

E Digitus-quintus-varus-Fehlstellungen sind i.d.R. erworben.

Eine der folgenden Aussagen ist falsch. Welche?
A Die Hallux-valgus-Deformität ist eine häufige Ursache für Kleinzehenfehlstellungen.

B Der Kleinzehenballen ist häufig mit einer Spreizfußkonfiguration vergesellschaftet.

C Rückfußfehlstellungen können Veränderungen der Kleinzehen verursachen.

D MTP-Luxationen zeigen sich häufig bei rheumatisch bedingten Fehlstellungen.

E Die Überlänge der II. Zehe zeigt sich häufig bei der sog. ägyptischen Fußform.
Nur eine der folgenden Aussagen ist korrekt. Welche?
A Das PIP-Gelenk ist wesentlich für Kleinzehenfehlstellungen verantwortlich.

B Die intrinsische Muskulatur ist ein wesentlicher passiver Stabilisator.

C Die intrinsische Muskulatur flektiert bei normalen anatomischen Verhältnissen im MTP-Gelenk.

D Die plantare Platte ist ein wesentlicher aktiver Stabilisator.

E Die Extensor-digitorum-longus-Sehne setzt am distalen Grundglied an.
Welche Aussage zur Klinik der Zehenfehlstellung ist falsch?
A Die Hammerzehe weist eine Beugestellung im PIP-Gelenk und eine Extension im MTP-Gelenk auf.

B Die Klauenzehe weist eine Beugestellung im MTP- und PIP-Gelenk auf.

C Die Endgelenkhammerzehe weist eine kontrakte oder flexible Beugefehlstellung im DIP-Gelenk auf.

D Der Kleinzehenballen schmerzt v.a. lateral.

E Die Crossover-Toe-Deformität betrifft meist die II. Zehe. 
Welche Aussage zur klinischen Untersuchung ist falsch?
A Die klinische Untersuchung umfasst immer beide Füße.

B Die Untersuchung umfasst die Palpation aller Zehengelenke.

C Bei flexiblen Kleinzehenfehlstellungen ist der Push-up-Test pathologisch.

D Eine vermehrte Translation kann Folge einer Verletzung der plantaren Platte sein.

E Endgelenkhammerzehen zeigen häufig Nagelwuchsstörungen und eine vermehrte Beschwielung.
Welche Aussage ist falsch? Die konservative Therapie von Kleinzehenfehlstellungen kann durch ...
A die Wahl des passenden, konfektionierten Schuhwerks erfolgen.

B eine Einlagenversorgung nach Maß erfolgen.

C eine professionelle Fußpflege unterstützt werden.

D physiotherapeutische Behandlung unterstützt werden.

E die Gipsredression der Kleinzehen erfolgen.
Was ist bei der OP-Vorbereitung zu berücksichtigen?
A Das Vorliegen einer peripheren arteriellen Verschlusskrankheit (pAVK) ist eine absolute Kontraindikation für die operative Korrektur von Kleinzehenfehlstellungen.

B Die Aufklärung allgemeiner und spezieller Risiken sollte nur das geplante Operationsverfahren beinhalten.

C Die operative Therapie ist der konservativen Behandlung immer vorzuziehen.

D Die Wahl des Operationsverfahrens bei Kleinzehenfehlstellung ist im Wesentlichen vom radiologischen Befund abhängig.

E Die Korrektur von Kleinzehenfehlstellungen kann zu einer Verbesserung der Weichteilverhältnisse führen.
Wodurch kann die operative Korrektur einer Krallenzehenfehlstellung nicht erfolgen?
A PIP-Arthrodese
B Flexor-digitorum-longus-Sehnentransfer
C Verlängerung der Extensor-digitorum-longus-Sehne
D distale Chevron-Osteotomie
E dorsale Kapsulotomie

Wodurch sind die Komplikationen nach operativer Kleinzehenkorrektur gekennzeichnet?
A Die postoperative Schwellung sollte durch Kühlung, Hochlagern und Schonung behandelt werden.

B Komplikationen nach operativer Kleinzehenkorrektur sind sehr häufig und führen meist zur Amputation der Zehe.

C Überkorrekturen sind häufiger als Unterkorrekturen.

D Pseudarthrosen sollten immer operativ revidiert werden.

E Einliegendes Osteosynthesematerial muss nach 6 Monaten entfernt werden.
Die Nachbehandlung von operativ behandelten Kleinzehendeformitäten ...
A ist nach 4 Wochen abgeschlossen.

B sollte durch redressierende Verbände und Orthesen erfolgen.

C sollte im Gips erfolgen.

D erlaubt aufgrund der fragilen Knochen keine Vollbelastung für 6 Wochen.

E erfordert immer die Verwendung von Unterarmgehstützen. 